\title{
Quantitative Game Semantics for Linear Logic
}

\author{
Ugo Dal Lago* $\quad$ Olivier Laurent ${ }^{\dagger}$
}

\begin{abstract}
We present a game-based semantic framework into which the time complexity of any IMELL proof can be read out of its interpretation. This gives a compositional view of the geometry of interaction framework introduced by the first author. In our model the time measure is given by means of slots, as introduced by Ghica in a recent paper. The cost associated to a strategy is polynomially related to the normalization time of the interpreted proof, in the style of a complexity-theoretical full abstraction result.
\end{abstract}

\section{Introduction}

Implicit computational complexity (ICC) is a very active research area lying at the intersection between mathematical logic, computational complexity and programming language theory. In the last years, a myriad of systems derived from mathematical logic (often through the Curry-Howard correspondence) and characterizing complexity classes (e.g. polynomial time, polynomial space or logarithmic space) have been proposed.

The techniques used to analyze ICC systems are usually ad-hoc and cannot be easily generalized to other (even similar) systems. Moreover, checking whether extending an existing ICC system with new constructs or new rules would break the correspondence with a given complexity class is usually not easy: soundness must be (re)proved from scratch. Take, for example, the case of subsystems of Girard's linear logic capturing complexity classes: there are at least three distinct subsystems of linear logic corresponding to polynomial time, namely bounded linear logic, light linear logic and soft linear logic. All of them can be obtained by properly restricting the rules governing the exponential connectives. Even if they have not been introduced independently, correspondence with polynomial time had to be reproved thrice. Moreover, it is not clear how robust the correspondence with polynomial time is. In other words, there is not any principled way to check whether extending one of the above systems with new logical rules would break (polytime) soundness. We need to understand why certain restrictions on the usual comonoidal exponential discipline in linear logic lead to characterizations of certain complexity classes.

This is the typical situation where semantics can be useful. And, indeed, some proposals for semantic frameworks into which some existing ICC systems can be interpreted have already appeared in the literature. Moreover, there are some proposals for semantic models in which the interpretation "reveals" quantitative, intensional, properties of proofs and programs. One of them [5] is due to the first author and is based on context semantics. There, the complexity of a proof is obtained by a global analysis of its interpretation as a set of paths.

In this paper, we show that the above mentioned context semantics can be put into a more interactive form by defining a game model for multiplicative and exponential linear logic and showing a quantitative correspondence between the interpretation of any proof and the time needed to normalize the proof.

Context semantics is a model of Girard's geometry of interaction. As a consequence, turning it into an AJM game model should not be difficult (at least in principle), due to the well-known

\footnotetext{
*Dipartimento di Scienze dell'Informazione, Università di Bologna. dallago@cs.unibo.it Partially supported by a Marie Curie EIF grant "ASFIX".

†Preuves Programmes Systèmes, CNRS - Université Paris 7. Olivier.Laurent@pps.jussieu.fr Partially supported by the French ANR "NO-CoST" project (JC05_43380).
} 


$$
\begin{gathered}
\frac{\Gamma \vdash A \quad \Delta, A \vdash B}{\varsigma \vdash A} A \quad \frac{\Gamma \vdash B}{\varsigma(\Gamma, \Delta) \vdash B} U \quad \frac{\Gamma, ! A, ! A \vdash B}{\varsigma(\Gamma, ! A) \vdash B} C \\
\frac{\Gamma, A \vdash B}{\varsigma(\Gamma) \vdash A \multimap B} R_{\multimap} \quad \frac{\Gamma \vdash A \quad \Delta, B \vdash C}{\varsigma(\Gamma, \Delta, A \multimap B) \vdash C} L_{-} \quad \frac{\Gamma \vdash A}{\varsigma(\Gamma, \Delta) \vdash A \otimes B} R_{\otimes} \quad \frac{\Delta, A, B \vdash C}{\varsigma(\Gamma, A \otimes B) \vdash C} L_{\otimes} \\
\frac{A_{1}, \ldots, A_{n} \vdash B}{\varsigma\left(! A_{1}, \ldots, ! A_{n}\right) \vdash ! B} P ! \quad \frac{\Gamma, A \vdash B}{\varsigma(\Gamma, ! A) \vdash B} D ! \quad \frac{\Gamma, ! ! A \vdash B}{\varsigma(\Gamma, ! A) \vdash B} N_{!}
\end{gathered}
$$

Figure 1: A sequent calculus for IMELL

strong correspondence between the two frameworks (see [3], for example). But there are at least two problems: first of all, the context semantics framework described in [5] is slightly different from the original one and, as such, it is not a model of geometry of interaction. This is why we introduced a lifting construction in our game model.

Moreover, the global analysis needed in [5] to extract the complexity of a proof from its interpretation cannot be easily turned into a more interactive analysis, in the spirit of game semantics. The extraction of time bounds from proof interpretations is somehow internalized here through the notion of time analyzer (see Section 2). One of the key technical lemmas towards the quantitative correspondence cited above is proved through a game-theoretical reducibility argument (see Section 4).

Another semantic framework designed with similar goals is Ghica's slot games [7]. There, however, the idea is playing slots in correspondence with any potential redex in a program, while here we focus on exponentials. On the other hand, the idea of using slots to capture intensional properties of proofs (or programs) in an interactive way is one of the key ingredients of this paper. In Section 5 the reader can find a more detailed comparison with Ghica's work. To keep the presentation simple, we preferred to adopt Ghica's way of introducing cost into games, rather than Leperchey's time monad.

In Baillot and Pedicini's geometry of interaction model [4], the "cost" of a proof is strongly related to the length of regular paths in its interpretation. But this way, one can easily define a family of terms which normalize in linear time but have exponential cost.

\section{Syntax}

We here introduce multiplicative exponential linear logic as a sequent calculus. It would be more natural to deal with proof-nets instead of the sequent calculus, but our semantic constructions will rely on a precise sequentiality in proof constructions that we would have to rebuild in a proof-net setting.

The language of formulas is defined by the following productions:

$$
A::=\alpha|A \multimap A| A \otimes A \mid ! A
$$

where $\alpha$ ranges over a countable set of atoms. A context is a sequence $\Gamma=A_{1}, \ldots, A_{n}$ of formulas. If $\Gamma=A_{1}, \ldots, A_{n}$ is a context and $\varsigma:\{1, \ldots, n\} \rightarrow\{1, \ldots, n\}$ is a permutation, then $\varsigma(\Gamma)$ stands for the context $A_{\varsigma(1)}, \ldots, A_{\varsigma(n)}$.

The rules in Figure 1 define a sequent calculus for (intuitionistic) multiplicative and exponential linear logic, IMELL, with an exchange rule integrated in the other ones.

Given any proof $\pi: \Gamma \vdash A$, we can build another proof $[\pi]: \Phi, \Gamma \vdash A$, where $\Phi$ is a sequence in the form $!^{k_{1}}\left(A_{1} \multimap A_{1}\right), \ldots, !^{k_{n}}\left(A_{n} \multimap A_{n}\right)$. We say that "cuts are exposed" in $[\pi]$. It is defined as follows, by induction on the structure of $\pi$ : 
- If the last rule in $\pi$ is not $U$ and the immediate subproofs of $\pi$ are $\rho_{1}, \ldots, \rho_{n}$, then $[\pi]$ is obtained from $\left[\rho_{1}\right], \ldots,\left[\rho_{n}\right]$ in the natural way. For a promotion rule, as an example, $\pi$ and $[\pi]$ are given by:

$$
\frac{\rho: A_{1}, \ldots, A_{n} \vdash B}{\varsigma\left(! A_{1}, \ldots, ! A_{n}\right) \vdash ! B} P_{!} \quad \frac{[\rho]: \Phi, A_{1}, \ldots, A_{n} \vdash B}{! \Phi, \varsigma\left(! A_{1}, \ldots, ! A_{n}\right) \vdash ! B} P_{!}
$$

- For a cut rule, $\pi$ and $[\pi]$ are given by:

$$
\frac{\rho: \Gamma \vdash B \quad \sigma: \Delta, B \vdash A}{\varsigma(\Gamma, \Delta) \vdash A} U \quad \frac{[\rho]: \Phi, \Gamma \vdash B \quad[\sigma]: \Psi, \Delta, B \vdash A}{\Phi, B \multimap B, \Psi, \varsigma(\Gamma, \Delta) \vdash A} L_{\multimap}
$$

If $\pi: \Gamma \vdash A$ is a proof and $[\pi]: \Phi, \Gamma \vdash A$, we can label formulas $!^{k}(B \multimap B)$ in $\Phi$ with labels reflecting the rules which introduced the two formula occurrences $B$. We explain this concept through an example. If $\pi$ is the following proof:

$$
\begin{aligned}
& \overline{\alpha \multimap \beta \vdash \alpha \multimap \beta}^{\alpha} \frac{\overline{\alpha \vdash \alpha}^{A-\beta \vdash \beta} \overline{\beta \vdash \beta}^{A}}{\alpha, \alpha \multimap \beta \vdash \beta} L_{-}
\end{aligned}
$$

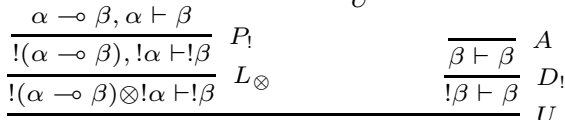

$$
\begin{aligned}
& !(\alpha \multimap \beta) \otimes ! \alpha \vdash \beta
\end{aligned}
$$

then $[\pi]$ will be the following proof:

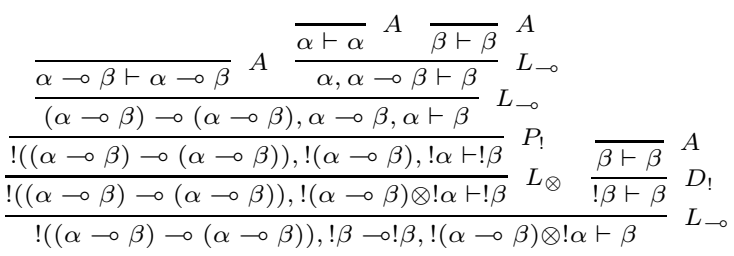

As expected, the conclusion of $[\pi]$ includes two formulas which are not in the conclusion of $\pi$, namely $!((\alpha \multimap \beta) \multimap(\alpha \multimap \beta))$ and $! \beta \multimap ! \beta$. The first one corresponds to the leftmost cut in $\pi$ : there, the two occurrences of $\alpha \multimap \beta$ are introduced by an axiom and by a $L_{\multimap}$ rule. So the natural label for it is $A-L_{-}$The second one corresponds to the rightmost cut in $\pi$ : the two occurrences of ! $\beta$ are introduced by $P_{!}$and $D_{!}$, respectively. As a consequence, the label for it is $P_{!}-D_{!}$. We obtain the following decoration of $[\pi]$ :

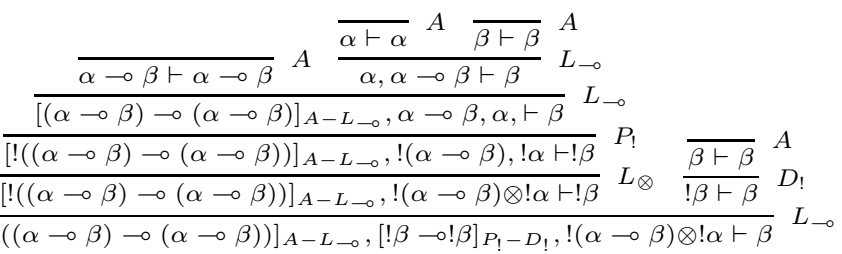

Cut-elimination The cut-elimination steps $\pi \rightsquigarrow \rho$ are an easy adaptation of the usual ones. We just have to take care of the exchange parts, but they can be handled without any particular problem.

$$
\begin{aligned}
& \frac{\frac{A_{1}, \ldots, A_{n} \vdash B}{\varsigma\left(! A_{1}, \ldots, ! A_{n}\right) \vdash ! B} P_{!} \frac{\frac{A_{1}, \ldots, A_{n} \vdash B}{\varsigma\left(! A_{1}, \ldots, ! A_{n}\right) \vdash ! B} P_{!} \quad \Gamma, ! B, ! B \vdash C}{\varsigma\left(! A_{1}, \ldots, ! A_{n}\right), \Gamma, ! B \vdash C}}{\frac{\Gamma, ! A_{1}, ! A_{1}, \ldots, ! A_{n}, ! A_{n} \vdash C}{! A_{n}, \Gamma, ! A_{1}, ! A_{1}, \ldots, ! A_{n-1}, ! A_{n-1} \vdash C}} C \\
& \frac{\frac{A_{1}, \ldots, A_{n} \vdash B}{\varsigma\left(! A_{1}, \ldots, ! A_{n}\right) \vdash ! B} P_{!} \frac{\Gamma, ! B, ! B \vdash C}{\vartheta(\Gamma), ! B \vdash C}}{\varpi\left(! A_{1}, \ldots, ! A_{n}, \Gamma\right) \vdash C} U \quad \rightsquigarrow \quad \frac{1}{\varpi\left(! A_{1}, \ldots, ! A_{n}, \Gamma\right) \vdash C} C
\end{aligned}
$$




$$
\begin{aligned}
& \stackrel{\frac{\Gamma \vdash C}{! A_{n}, \Gamma \vdash C} W}{W} \\
& \frac{\frac{A_{1}, \ldots, A_{n} \vdash B}{\varsigma\left(! A_{1}, \ldots, ! A_{n}\right) \vdash ! B} \quad P ! \frac{\Gamma \vdash C}{\vartheta(\Gamma), ! B \vdash C}}{\varpi\left(! A_{1}, \ldots, ! A_{n}, \Gamma\right) \vdash C} W \\
& \rightsquigarrow \quad \frac{\frac{}{! A_{2} \ldots, ! A_{n}, \Gamma \vdash C}}{\varpi\left(! A_{1}, \ldots, ! A_{n}, \Gamma\right) \vdash C} W \\
& \begin{array}{l}
\frac{A_{1}, \ldots, A_{n} \vdash B \quad \Gamma, B \vdash C}{\Gamma, A_{1}, \ldots, A_{n} \vdash C} U \\
\frac{!_{n}, \Gamma, A_{1}, \ldots, A_{n-1} \vdash C}{D !} \\
D !
\end{array} \\
& \frac{\frac{A_{1}, \ldots, A_{n} \vdash B}{\varsigma\left(! A_{1}, \ldots, ! A_{n}\right) \vdash ! B} P_{!} \frac{\Gamma, B \vdash C}{\vartheta(\Gamma), ! B \vdash C}}{\varpi\left(! A_{1}, \ldots, ! A_{n}, \Gamma\right) \vdash C} D \\
& \begin{array}{cc}
\frac{\vdots}{! A_{2}, \ldots, ! A_{n}, \Gamma, A_{1} \vdash C} & D ! \\
\varpi\left(! A_{1}, \ldots, ! A_{n}, \Gamma\right) \vdash C & D !
\end{array} \\
& \frac{A_{1}, \ldots, A_{n} \vdash B}{! A_{1}, \ldots, ! A_{n} \vdash ! B} \\
& \frac{\frac{\frac{1 A_{1}, \ldots, ! ! A_{n} \vdash ! ! B}{} P_{!} \quad \Gamma, ! ! B \vdash C}{\Gamma, ! ! A_{1}, \ldots, ! ! A_{n} \vdash C}}{\frac{! A_{n}, \Gamma, ! ! A_{1}, \ldots, ! ! A_{n-1} \vdash C}{} N_{!}} N_{!} \\
& \frac{\frac{A_{1}, \ldots, A_{n} \vdash B}{\varsigma\left(! A_{1}, \ldots, ! A_{n}\right) \vdash ! B} \quad P_{!} \frac{\Gamma, ! ! B \vdash C}{\vartheta(\Gamma), ! B \vdash C}}{\varpi\left(! A_{1}, \ldots, ! A_{n}, \Gamma\right) \vdash C} U \\
& \rightsquigarrow \quad \frac{\overline{! A_{2}, \ldots, ! A_{n}, \Gamma, ! ! A_{1} \vdash C}}{\varpi\left(! A_{1}, \ldots, ! A_{n}, \Gamma\right) \vdash C} N_{!} \\
& \frac{\frac{A_{1}, \ldots, A_{n} \vdash B}{\varsigma\left(! A_{1}, \ldots, ! A_{n}\right) \vdash ! B} P_{!} \frac{\vartheta\left(B_{1}, \ldots, B_{m}, B\right) \vdash C}{! B_{1}, \ldots, ! B_{m}, ! B \vdash ! C}}{\varpi\left(! A_{1}, \ldots, ! A_{n}, ! B_{1}, \ldots, ! B_{m}\right) \vdash ! C} P_{!} \quad \rightsquigarrow \quad \frac{\frac{A_{1}, \ldots, A_{n} \vdash B \quad \varphi\left(B_{1}, \ldots, B_{m}\right), B \vdash C}{\vartheta\left(B_{1}, \ldots, B_{m},\left\langle A_{1}, \ldots, A_{n}\right\rangle\right) \vdash C}}{\varpi\left(! A_{1}, \ldots, ! A_{n}, ! B_{1}, \ldots, ! B_{m}\right) \vdash ! C} P_{!}
\end{aligned}
$$

where $\vartheta\left(B_{1}, \ldots, B_{m},\left\langle A_{1}, \ldots, A_{n}\right\rangle\right)$ is $B_{\vartheta(1)}, \ldots, B_{\vartheta(k-1)}, A_{1}, \ldots, A_{n}, B_{\vartheta(k+1)}, \ldots, B_{\vartheta(m)}$ if $\vartheta^{-1}(m+$ $1)=k$. To avoid stupid loops, we allow a cut rule $c$ to commute upwards with another cut rule $d$ during reduction only if $d$ introduces the left premise of $c$ (and not if $d$ introduces the right premise of $c$ ).

For our complexity analysis to make sense, we will restrict the cut elimination procedure to a particular strategy of reduction called surface reduction. From a proof-net point of view it corresponds to reducing cuts at depth 0 only. In a sequent calculus setting, we only apply a reduction step to a cut rule if it is not above a promotion rule $P_{\text {! }}$.

For practical reasons, we introduce a particular atomic formula $\mathbb{U}$ and we extend the IMELL system with the following "pseudo"-rules (which are not valid from a logical point of view):

$$
\overline{\vdash X} a \quad \frac{\Gamma \vdash A}{\varsigma(X, \Gamma) \vdash A} w
$$

where $X$ is any atomic formula: $\alpha$ or $\mathbb{U}$.

This allows us to define a proof $\mathrm{TA}_{A}$ of $A \vdash \mathbb{U}$ and a proof $\mathrm{TA}_{A}^{0}$ of $\vdash A$. $\mathrm{TA}_{A}$ is called the time analyzer of $A$. They are defined by mutual induction on $A$ :

$$
\begin{aligned}
& \frac{\overline{\vdash \mathbb{U}}^{a}}{\mathrm{TA}_{\alpha}: \alpha \vdash \mathbb{U}} w \quad \overline{\mathrm{TA}_{\alpha}^{0}: \vdash \alpha} a \\
& \frac{\mathrm{TA}_{A}: A \vdash \mathbb{U} \frac{\mathrm{TA}_{B}: B \vdash \mathbb{U}}{\mathbb{U}, B \vdash \mathbb{U}}}{\frac{A, B \vdash \mathbb{U}}{\mathrm{TA}_{A \otimes B}: A \otimes B \vdash \mathbb{U}} L_{\otimes}} U \\
& \frac{\mathrm{TA}_{A}^{0}: \vdash A \quad \mathrm{TA}_{B}: B \vdash \mathbb{U}}{\mathrm{TA}_{A \multimap B}: A \multimap B \vdash \mathbb{U}} L_{\multimap} \\
& \frac{\mathrm{TA}_{A}^{0}: \vdash A \quad \mathrm{TA}_{B}^{0}: \vdash B}{\mathrm{TA}_{A \otimes B}^{0}: \vdash A \otimes B} R_{\otimes} \\
& \frac{\mathrm{TA}_{A}: A \vdash \mathbb{U} \frac{\mathrm{TA}_{B}^{0}: \vdash B}{\mathbb{U} \vdash B}}{\frac{A \vdash B}{\mathrm{TA}_{A \multimap B}^{0}: \vdash A \multimap B}}= \\
& \frac{\overline{\vdash \mathbb{U}}^{a}}{\mathrm{TA}_{! A}: ! A \vdash \mathbb{U}} W \\
& \frac{\mathrm{TA}_{A}^{0}: \vdash A}{\mathrm{TA}_{! A}^{0}: \vdash ! A} P !
\end{aligned}
$$




\section{Game Semantics}

The game model we use is based on the constructions presented in [1]. We extend it with the simplest (and well known) exponential construction (by enriching moves with copy indexes) together with a lifting operation (adding two fresh moves at the beginning of a game).

\subsection{Games}

A game $A$ consists in:

- A set of moves $M_{A}$. $\sqsubseteq_{A}$ is the prefix relation over $M_{A}^{*}$, i.e., $s \sqsubseteq_{A} r$ iff $s$ is a prefix of $r$. $\sqsubseteq$ takes the place of $\sqsubseteq_{A}$ when the underlying game $A$ is clear from the context.

- A function $\lambda_{A}: M_{A} \rightarrow\{\mathrm{P}, \mathrm{O}\}$. $\overline{\lambda_{A}}$ denotes the function from $M_{A}$ to $\{\mathrm{P}, \mathrm{O}\}$ defined by $\overline{\lambda_{A}}(m) \neq \lambda_{A}(m) . \quad M_{A}^{\circledast}$ denotes the subset of $M_{A}^{*}$ containing alternated, opponent-initiated sequences only, i.e., $\lambda_{A}(m)=\mathrm{O}$ whenever $m s \in M_{A}^{\circledast}$ and, moreover, $\lambda_{A}(m) \neq \lambda_{A}(n)$ whenever smnr $\in M_{A}^{\circledast}$. $M_{A}^{\mathrm{P}}$ and $M_{A}^{\mathrm{O}}$ are subsets of $M_{A}$ defined in the natural way.

- A set $P_{A}$ of valid plays such that $P_{A} \subseteq M_{A}^{\circledast}$ and $P_{A}$ is closed under prefixes.

The language $\mathscr{E}$ of exponential signatures is defined by induction from the following set of productions: $\quad t, s, u::=\mathrm{f}|\mathrm{e}| \mathrm{I}(t)|\mathrm{r}(t)| \mathrm{p}(t) \mid \mathrm{n}(t, t)$.

\subsection{Constructions on Games}

To each connective corresponds a game construction. In the particular case of the exponential connective !, we decompose its interpretation in our model into a "sequentiality construction" given by lifting and a "copying construction" given by a traditional exponential construction with copy indexes given by exponential signatures.

\section{- Atomic game $\alpha$ :}

- $M_{\alpha}=\left\{\alpha^{\mathrm{P}}, \alpha^{\mathrm{O}}\right\}$.

- $\lambda_{\alpha}\left(\alpha^{\mathrm{P}}\right)=\mathrm{P}$ and $\lambda_{\alpha}\left(\alpha^{\mathrm{O}}\right)=\mathrm{O}$.

- $P_{\alpha}$ is $\left\{\varepsilon, \alpha^{\mathrm{O}}, \alpha^{\mathrm{O}} \cdot \alpha^{\mathrm{P}}\right\}$.

One particular atomic game is called $\mathbb{U}$ with moves denoted by a (instead of $\alpha^{\mathrm{P}}$ ) and $\mathrm{q}($ instead of $\left.\alpha^{\mathrm{O}}\right)$.

- Tensor game $A \otimes B$ :

- $M_{A \otimes B}=M_{A}+M_{B}$. If $s \in M_{A \otimes B}^{*}$, then $s_{A}$ denotes the subsequence of $s$ consisting of moves in $M_{A}$. Similarly for $s_{B}$.

- $\lambda_{A \otimes B}=\lambda_{A}+\lambda_{B}$.

- The elements of $P_{A \otimes B}$ are sequences $s \in M_{A \otimes B}^{\circledast}$ such that $s_{A} \in P_{A}, s_{B} \in P_{B}$.

- Arrow game $A \multimap B$ :

- $M_{A \multimap B}=M_{A}+M_{B}$.

- $\lambda_{A \multimap B}=\overline{\lambda_{A}}+\lambda_{B}$.

- The elements of $P_{A \multimap B}$ are sequences $s \in M_{A \multimap B}^{\circledast}$ such that $s_{A} \in P_{A}, s_{B} \in P_{B}$.

- Lifting game $\downarrow A$ :

- $M_{\downarrow A}=M_{A}+\{$ open, close $\}$.

- $\lambda_{\downarrow A}(m)=\lambda_{A}(m)$ whenever $m \in M_{A}, \lambda_{\downarrow A}$ (open) $=\mathrm{O}, \lambda_{\downarrow A}$ (close) $=\mathrm{P}$.

- $P_{\downarrow A}$ is $\{\varepsilon$, open $\} \cup\left\{\right.$ open $\cdot$ close $\left.\cdot s \mid s \in P_{A}\right\}$.

- Exponential game \#A:

- $M_{\# A}=\mathscr{E} \times M_{A}$. Given any sequence $s$ in $M_{\# A}^{*}$ and any exponential signature $t, s_{t}$ denotes the subsequence of $s$ consisting in moves in the form $(t, m)$. Given any sequence $s$ in $M_{A}^{*}$ and any exponential signature $t, t \times s$ denotes the sequence in $M_{\# A}^{*}$ obtained by pairing each move in $s$ with $t$.

- $\lambda_{\# A}(t, m)=\lambda_{A}(m)$.

- The elements of $P_{\# A}$ are sequences $s \in M_{\# A}^{\circledast}$ such that for every $t \in \mathscr{E}, s_{t}=t \times r$ with $r \in P_{A}$.

We will often use the notation $! A$ for $\# \downarrow A$. 


\section{3 $\quad$ Strategies}

Proofs are interpreted as particular strategies over games. However since we are not looking for full completeness results (but for complexity full abstraction instead), we are not particularly restrictive on the kind of strategies we deal with. There is no particular notion of uniformity on strategies such as history-freeness, innocence, etc. Important properties of strategies coming from proofs will be recovered through realizability (see Section 4).

A strategy $\sigma$ over a game $A$ is a non-empty set of even-length plays in $P_{A}$ satisfying the following conditions:

- $\sigma$ is even-prefix-closed;

- $\sigma$ is deterministic: if smn $\in \sigma$, smo $\in \sigma$, then $n=o$.

A strategy $\sigma$ over $A$ is total if $s \in \sigma$ and $s m \in P_{A}$ implies $s m n \in \sigma$ for some $n \in M_{A}$.

Composition of strategies can be defined in the usual way. Given a strategy $\sigma$ over $A \multimap B$ and $\tau$ over $B \multimap C$, we can first define $\sigma \| \tau$ as follows:

$$
\sigma \| \tau=\left\{s \in\left(M_{A} \cup M_{B} \cup M_{C}\right)^{*} \mid s_{A, B} \in \sigma \wedge s_{B, C} \in \tau\right\} .
$$

The composition of $\sigma$ and $\tau$, denoted $\sigma ; \tau$ is simply $\sigma ; \tau=\left\{s_{A, C} \mid s \in \sigma \| \tau\right\}$.

Proposition 1 If $\sigma$ is a strategy over $A \multimap B$ and $\tau$ is a strategy over $B \multimap C$, then $\sigma$; $\tau$ is a strategy over $A \multimap C$.

A useful restriction on strategies is given by history-free strategies $\sigma$ satisfying: if sm next $_{\sigma}(m) \in$ $P_{A}$ then $s m \cdot \operatorname{next}_{\sigma}(m) \in \sigma$ if and only if $s \in \sigma$ where ext $_{\sigma}$ is the generating partial function from $M_{A}^{\mathrm{O}}$ to $M_{A}^{\mathrm{P}}$. The composition of two history-free strategies is an history-free strategy generated by the composition of generating functions. Some of the strategies we use happen to be history-free, but not all of them are.

The history-free identity strategy $\mathbf{i d}_{A}$ over $A \multimap A$ is given by the generating function (assume $A \multimap A$ is $\left.A_{1} \multimap A_{2}\right):$

$$
\begin{aligned}
& \forall m \in M_{A}^{\mathrm{O}} \cdot \text { next }_{\mathbf{i d}_{A}}\left(m_{A_{2}}\right)=m_{A_{1}} \\
& \forall m \in M_{A}^{\mathrm{P}} \cdot \text { next }_{\mathbf{i d}_{A}}\left(m_{A_{1}}\right)=m_{A_{2}}
\end{aligned}
$$

According to [1], games and strategies define a symmetric monoidal closed category (SMCC).

\subsection{Constructions on Strategies}

We describe elementary constructions on strategies which, once plug together, will allow us to interpret proofs in the game model.

- Left-lifting Strategy: Given a strategy $\sigma$ over the game $A \otimes B \multimap C$, the subset $\operatorname{ll}(\sigma)$ of $P_{\downarrow A \otimes B \multimap C}$ is defined as follows:

$$
\mathbf{l l}(\sigma)=\{\varepsilon\} \cup\left\{m \cdot \text { open }_{\downarrow A} \mid \exists m s \in \sigma\right\} \cup\left\{m \cdot \text { open }_{\downarrow A} \cdot \text { close }_{\downarrow A} \cdot s \mid m s \in \sigma\right\}
$$

In the same spirit, we can define $\mathbf{l l}_{B}(\sigma)$ over $A \otimes \downarrow B \multimap C$ (so that $\mathbf{l l}_{A}(\sigma)=\mathbf{l l}(\sigma)$ ).

- Right-lifting Strategy: Given a strategy $\sigma$ over the game $A$, the subset $\operatorname{rl}(\sigma)$ of $P_{\downarrow A}$ is defined as follows:

$$
\operatorname{rl}(\sigma)=\{\varepsilon\} \cup\left\{\text { open }_{\downarrow A} \cdot \text { close }_{\downarrow A} \cdot s \mid s \in \sigma\right\}
$$

Using the immediate bijection between $M_{\downarrow(A \multimap B)}$ and $M_{A \multimap \downarrow}$, if $\sigma$ is a strategy over $A \multimap B$, we will often use $\mathbf{r l}(\sigma)$ as a strategy over $A \multimap \downarrow B$.

- Lifting Strategy: Given a strategy $\sigma$ over the game $A_{1} \otimes \cdots \otimes A_{n} \multimap B$, the subset $\mathbf{l}(\sigma)$ of $P_{\downarrow A_{1} \otimes \cdots \otimes \downarrow A_{n} \multimap \downarrow B}$ is defined by $\mathbf{l}(\sigma)=\mathbf{l l}_{A_{1}}\left(\ldots \mathbf{l}_{A_{n}}(\mathbf{r l}(\sigma))\right)$.

- Dereliction Strategy: The subset $\mathbf{d}_{A}$ of $P_{\# A \multimap A}$ is the one induced by the following (assume $\# A \multimap A$ is $\left.\# A_{1} \multimap A_{2}\right)$ :

$$
\begin{aligned}
\forall m \in M_{A}^{\mathrm{O}} \cdot \operatorname{next}_{\mathbf{d}_{A}}\left(m_{A_{2}}\right) & =(\mathrm{e}, m)_{\# A_{1}} \\
\forall m \in M_{A}^{\mathrm{P}} \cdot \operatorname{next}_{\mathbf{d}_{A}}\left((\mathrm{e}, m)_{\# A_{1}}\right) & =m_{A_{2}}
\end{aligned}
$$


- Weakening Strategy: The subset $\mathbf{w}_{A}$ of $P_{\# A-A}$ is the one induced by the following (assume $\# A \multimap A$ is $\left.\# A_{1} \multimap A_{2}\right)$ :

$$
\begin{aligned}
\forall m \in M_{A}^{\mathrm{O}} \cdot \operatorname{next}_{\mathbf{w}_{A}}\left(m_{A_{2}}\right) & =(\mathrm{f}, m)_{\# A_{1}} \\
\forall m \in M_{A}^{\mathrm{P}} \cdot \operatorname{next}_{\mathbf{w}_{A}}\left((\mathrm{f}, m)_{\# A_{1}}\right) & =m_{A_{2}}
\end{aligned}
$$

In spite of their similarity, the $\mathbf{d}_{A}$ and $\mathbf{w}_{A}$ strategies will be used in a quite different manner for interpreting proofs.

- Digging Strategy: The subset $\mathbf{n}_{A}$ of $P_{\# \downarrow A-\downarrow \# \downarrow \# \downarrow A}$ is the one induced by the following (assume $\# \downarrow A \multimap \downarrow \# \downarrow \# \downarrow A$ is $\# \downarrow A_{1} \multimap \downarrow \# \downarrow \# \downarrow A_{2}$ ):

$$
\begin{aligned}
\operatorname{next}_{\mathbf{n}_{A}}\left(\text { open }_{\downarrow \# \downarrow \# \downarrow A_{2}}\right) & =(\mathrm{f}, \text { open })_{\# \downarrow A_{1}} \\
\operatorname{next}_{\mathbf{n}_{A}}\left((\mathrm{f}, \text { close })_{\# \downarrow A_{1}}\right) & =\text { close }_{\downarrow \# \downarrow \# \downarrow A_{2}} \\
\operatorname{next}_{\mathbf{n}_{A}}\left((t, \text { open })_{\downarrow \# \downarrow \# \downarrow A_{2}}\right) & =(\mathrm{p}(t), \text { open })_{\# \downarrow A_{1}} \\
\operatorname{next}_{\mathbf{n}_{A}}\left((\mathrm{p}(t), \text { close })_{\# \downarrow A_{1}}\right) & =(t, \text { close })_{\downarrow \# \downarrow \# \downarrow A_{2}} \\
\forall m \in M_{A}^{\mathrm{O}} \cdot \operatorname{next}_{\mathbf{n}_{A}}\left((t,(s, m))_{\downarrow \# \downarrow \# \downarrow A_{2}}\right) & =(\mathrm{n}(t, s), m)_{\# \downarrow A_{1}} \\
\forall m \in M_{A}^{\mathrm{P}} \cdot \operatorname{next}_{\mathbf{n}_{A}}\left((\mathrm{n}(t, s), m)_{\# \downarrow A_{1}}\right) & =(t,(s, m))_{\downarrow \# \downarrow \# \downarrow A_{2}}
\end{aligned}
$$

- Contraction Strategy: The subset $\mathbf{c}_{A}$ of $P_{\# \downarrow A \multimap \downarrow \# \downarrow A \otimes \# \downarrow A}$ is the one induced by the following (assume $\# \downarrow A \multimap \downarrow \# \downarrow A \otimes \# \downarrow A$ is $\# \downarrow A_{1} \multimap \downarrow \# \downarrow A_{2} \otimes \# \downarrow A_{3}$ ):

$$
\begin{aligned}
\operatorname{next}_{\mathbf{c}_{A}}\left(\text { open }_{\downarrow \# \downarrow A_{2}}\right) & =\left(\mathrm{f}, \text { open }_{\# \downarrow A_{1}}\right. \\
\operatorname{next}_{\mathbf{c}_{A}}\left(\left(\mathrm{f}, \text { close }_{\# \downarrow \downarrow A_{1}}\right)\right. & =\text { close }_{\downarrow \# \downarrow A_{2}} \\
\forall m \in M_{A}^{\mathrm{O}} \cdot \operatorname{next}_{\mathbf{c}_{A}}\left((t, m)_{\downarrow \# \downarrow A_{2}}\right) & =(\mathrm{I}(t), m)_{\# \downarrow A_{1}} \\
\forall m \in M_{A}^{\mathrm{P}} \cdot \operatorname{next}_{\mathbf{c}_{A}}\left((\mathrm{I}(t), m)_{\# \downarrow A_{1}}\right) & =(t, m)_{\downarrow \# \downarrow A_{2}} \\
\forall m \in M_{A}^{\mathrm{O}} \cdot \operatorname{next}_{\mathbf{c}_{A}}\left((t, m)_{\# \downarrow A_{3}}\right) & =(\mathrm{r}(t), m)_{\# \downarrow A_{1}} \\
\forall m \in M_{A}^{\mathrm{P}} \cdot \operatorname{next}_{\mathbf{c}_{A}}\left((\mathrm{r}(t), m)_{\# \downarrow A_{1}}\right) & =(t, m)_{\# \downarrow A_{3}}
\end{aligned}
$$

- Promotion Strategy: Given a strategy $\sigma$ over the game $A_{1} \otimes \cdots \otimes A_{n} \multimap B$, the subset $\mathbf{p}(\sigma)$ of $P_{\# A_{1} \otimes \cdots \otimes \# A_{n}-\# B}$ is defined as follows:

$$
\mathbf{p}(\sigma)=\left\{s \in P_{\# A_{1} \otimes \cdots \otimes \# A_{n} \multimap \# B} \mid \forall t . \exists r \in \sigma . s_{t}=t \times r\right\}
$$

We use the notation $\mathbf{p l}(\sigma)$ for $\mathbf{p}(\mathbf{l}(\sigma))$.

Proposition 2 For any game $A, \mathbf{d}_{A}, \mathbf{w}_{A}, \mathbf{n}_{A}$ and $\mathbf{c}_{A}$ are strategies. Let $\sigma$ be a strategy over $A_{1} \otimes \cdots \otimes A_{n} \multimap B$. Then $\mathbf{r l}(\sigma)$ and $\mathbf{p}(\sigma)$ are strategies and, if $n \geq 1, \operatorname{ll}(\sigma)$ is a strategy.

Proof. We can proceed as follows:

- The history-free set of plays $\sigma$ over $A$ inductively generated by a partial function $n e x t_{\sigma}$ :

$$
\begin{aligned}
\varepsilon & \in \sigma \\
s m \cdot \operatorname{ext}_{\sigma}(m) & \in \sigma \quad \text { if } s \in \sigma \text { and } s m \cdot \operatorname{next}_{\sigma}(m) \in P_{A}
\end{aligned}
$$

is always a strategy: it is non-empty, it contains only even-length plays in $P_{A}$, it is even-prefixclosed, and it is deterministic since next ${ }_{\sigma}$ is a function.

As a consequence, $\mathbf{d}_{A}, \mathbf{w}_{A}, \mathbf{n}_{A}$ and $\mathbf{c}_{A}$ are strategies.

- The lifting constructions $\operatorname{rl}(\sigma)$ and $\mathbf{l l}(\sigma)$ on strategies are coming from those given in $[8$, Chapter 3] which induce a structure of strong monad. For example:

$$
\mathbf{l}(\sigma)=\downarrow A_{1} \otimes A_{2} \otimes \cdots \otimes A_{n} \stackrel{\text { str }}{\longrightarrow} \downarrow\left(A_{1} \otimes \cdots \otimes A_{n}\right) \stackrel{[\sigma]}{\longrightarrow} B
$$

where $\mathbf{s t r}$ is the strength of the monad (or the identity if $n=1$ ).

- $\mathbf{p}(\sigma)$ is a strategy as given in the literature $[2,8]$. 


\subsection{Properties of Strategies}

We give here a list of the key properties we need about the constructions given above.

- If $\sigma: A_{0} \multimap A$ and $\tau: A \multimap B$ then $\sigma ; \operatorname{rl}(\tau)=\operatorname{rl}(\sigma ; \tau)$.

- If $\sigma: A_{0} \multimap A$ and $\tau: A \otimes B \multimap C$ then $\left(\sigma \otimes \mathbf{i d}_{\downarrow B}\right) ; \mathbf{l l}_{B}(\tau)=\mathbf{l l}_{B}\left(\left(\sigma \otimes \mathbf{i d}{ }_{B}\right) ; \tau\right)$.

- If $\sigma: A_{0} \multimap A, \delta: B_{0} \multimap B$ and $\tau: A \otimes B \multimap C$ then $(\operatorname{rl}(\sigma) \otimes \delta) ; \mathbf{l l}(\tau)=(\sigma \otimes \delta) ; \tau$ (in the particular case where $\tau$ does not contain any move in $A$, this is equal to $\delta ; \tau)$.

- If $\sigma: A \otimes B \multimap \downarrow C, \delta: D_{0} \multimap D$ and $\tau: C \otimes D \multimap E$ then $(\mathbf{l l}(\sigma) \otimes \delta) ; \operatorname{ll}(\tau)=\mathbf{l l}((\sigma \otimes \delta) ; \operatorname{ll}(\tau))$.

- If $\sigma: A_{1} \otimes \cdots \otimes A_{n} \multimap A$ and $\tau: A \otimes A_{n+1} \otimes \cdots \otimes A_{m} \multimap B$ then $\left(\mathbf{p}(\sigma) \otimes \mathbf{i d} \# A_{n+1} \otimes \cdots \otimes\right.$ $\left.\mathbf{i d}_{\# A_{m}}\right) ; \mathbf{p}(\tau)=\mathbf{p}\left(\left(\sigma \otimes \mathbf{i d}_{A_{n+1}} \otimes \cdots \otimes \mathbf{i d}_{A_{m}}\right) ; \tau\right)$.

- If $\sigma: A_{1} \otimes \cdots \otimes A_{n} \multimap B$ then $\mathbf{p}(\sigma) ; \mathbf{d}_{B}=\left(\mathbf{d}_{A_{1}} \otimes \cdots \otimes \mathbf{d}_{A_{n}}\right) ; \sigma\left(\right.$ and $\mathbf{p}(\sigma) ; \mathbf{w}_{B}=\left(\mathbf{w}_{A_{1}} \otimes \cdots \otimes\right.$ $\left.\left.\mathbf{w}_{A_{n}}\right) ; \sigma\right)$.

- If $\sigma: A_{1} \otimes \cdots \otimes A_{n} \multimap B$ then $\mathbf{p l}(\sigma) ; \mathbf{c}_{B}=\left(\mathbf{c}_{A_{1}} \otimes \cdots \otimes \mathbf{c}_{A_{n}}\right) ; \mathbf{l}(\mathbf{p l}(\sigma)) \otimes \mathbf{p l}(\sigma)$ (up to some permutation in the second composition turning $\downarrow ! A_{1} \otimes ! A_{1} \otimes \cdots \otimes \downarrow ! A_{n} \otimes ! A_{n}$ into $\downarrow ! A_{1} \otimes \cdots \otimes$ $\left.\downarrow ! A_{n} \otimes ! A_{1} \otimes \cdots \otimes ! A_{n}\right)$.

- If $\sigma: A_{1} \otimes \cdots \otimes A_{n} \multimap B$ then $\mathbf{p l}(\sigma) ; \mathbf{n}_{B}=\left(\mathbf{n}_{A_{1}} \otimes \cdots \otimes \mathbf{n}_{A_{n}}\right) ; \mathbf{l}(\mathbf{p l}(\mathbf{p l}(\sigma)))$.

\subsection{Interpretation of Proofs}

We define the strategy $\llbracket \pi \rrbracket$ interpreting a proof $\pi$.

The multiplicative rules are interpreted according to the symmetric monoidal closed structure of the category of games and strategies. The interpretation of the exponential rules is based on the constructions described above.

- Weakening: if $\sigma$ is a strategy over $\Gamma \multimap B$, it is also a strategy over $A \otimes \Gamma \multimap B$ and we can build $\left(\mathbf{w}_{\downarrow A} \otimes \mathbf{i d}_{\Gamma}\right) ; \mathbf{l l}(\sigma)$ as a strategy over $! A \otimes \Gamma \multimap B$.

- Contraction: if $\sigma$ is a strategy over $! A \otimes ! A \otimes \Gamma \multimap B$, we can build $\left(\mathbf{c}_{A} \otimes \mathbf{i d}_{\Gamma}\right) ; \mathbf{l l}(\sigma)$ as a strategy over $! A \otimes \Gamma \multimap B$.

- Promotion: if $\sigma$ is a strategy over $A_{1} \otimes \cdots \otimes A_{n} \multimap B$, we can build $\mathbf{p l}(\sigma)$ as a strategy over $! A_{1} \otimes \cdots \otimes ! A_{n} \multimap ! B$.

- Dereliction: if $\sigma$ is a strategy over $A \otimes \Gamma \multimap B$, we can build $\left(\mathbf{d}_{\downarrow} \otimes \otimes \mathbf{i d} \Gamma\right) ; \operatorname{ll}(\sigma)$ as a strategy over $! A \otimes \Gamma \multimap B$.

- Digging: if $\sigma$ is a strategy over !! $A \otimes \Gamma \multimap B$, we can build $\left(\mathbf{n}_{A} \otimes \mathbf{i d} \mathbf{d}_{\Gamma}\right) ; \mathbf{l l}(\sigma)$ as a strategy over $! A \otimes \Gamma \multimap B$.

Theorem 1 (Soundness) If $\pi \rightsquigarrow \rho$ then $\llbracket \pi \rrbracket=\llbracket \rho \rrbracket$.

Proof. The multiplicative steps are given by the SMCC structure. The permutations of formulas are handled by the symmetry of the SMCC structure. Since the key properties are given in the previous section, we consider only a few cases:

- Contraction: If $\sigma: A_{1} \otimes \cdots \otimes A_{n} \multimap B$ and $\tau: ! B \otimes ! B \otimes \Gamma \multimap C$, we have:

$$
\begin{aligned}
& \left(\mathbf{p l}(\sigma) \otimes \mathbf{i d}_{\Gamma}\right) ;\left(\left(\mathbf{c}_{B} \otimes \mathbf{i d}_{\Gamma}\right) ; \mathbf{l l}(\tau)\right) \\
& =\left(\left(\mathbf{p l}(\sigma) ; \mathbf{c}_{B}\right) \otimes \mathbf{i d}_{\Gamma}\right) ; \mathbf{l l}(\tau) \\
& =\left(\left(\left(\mathbf{c}_{A_{1}} \otimes \cdots \otimes \mathbf{c}_{A_{n}}\right) ; \mathbf{l}(\mathbf{p l}(\sigma)) \otimes \mathbf{p l}(\sigma)\right) \otimes \mathbf{i d}_{\Gamma}\right) ; \mathbf{l l}(\tau) \\
& =\left(\left(\mathbf{c}_{A_{1}} \otimes \cdots \otimes \mathbf{c}_{A_{n}}\right) \otimes \mathbf{i d}_{\Gamma}\right) ;\left(\mathbf{l}(\mathbf{p l}(\sigma)) \otimes \mathbf{p l}(\sigma) \otimes \mathbf{i d}_{\Gamma}\right) ; \mathbf{l l}(\tau) \\
& =\left(\mathbf{c}_{A_{1}} \otimes \cdots \otimes \mathbf{c}_{A_{n}} \otimes \mathbf{i d}_{\Gamma}\right) ;\left(\mathbf{l l}_{! A_{1}}\left(\ldots \mathbf{l}_{! A_{n}}\left(\left(\mathbf{r l}(\mathbf{p l}(\sigma)) \otimes \mathbf{p l}(\sigma) \otimes \mathbf{i d}_{\Gamma}\right) ; \mathbf{l l}(\tau)\right)\right)\right) \\
& =\left(\mathbf{c}_{A_{1}} \otimes \cdots \otimes \mathbf{c}_{A_{n}} \otimes \mathbf{i d}_{\Gamma}\right) ;\left(\mathbf{l l}_{! A_{1}}\left(\ldots \mathbf{l}_{! A_{n}}\left(\left(\mathbf{p l}(\sigma) \otimes \mathbf{p l}(\sigma) \otimes \mathbf{i d}_{\Gamma}\right) ; \tau\right)\right)\right) \\
& =\left(\mathbf{c}_{A_{1}} \otimes \cdots \otimes \mathbf{c}_{A_{n-1}} \otimes \mathbf{i d}_{! A_{n}} \otimes \mathbf{i d}_{\Gamma}\right) ;\left(\mathbf{i d}_{\downarrow ! A_{1}} \otimes \mathbf{i d}_{! A_{1}} \otimes \cdots \otimes \mathbf{i d}_{\downarrow ! A_{n-1}} \otimes \mathbf{i d}_{! A_{n-1}} \otimes \mathbf{c}_{A_{n}} \otimes \mathbf{i d}_{\Gamma}\right) ; \\
& \left(\mathbf{l l}_{! A_{1}}\left(\ldots \mathbf{l l}_{!_{A_{n}}}\left(\left(\mathbf{p l}(\sigma) \otimes \mathbf{p l}(\sigma) \otimes \mathbf{i d}_{\Gamma}\right) ; \tau\right)\right)\right) \\
& =\left(\mathbf{c}_{A_{1}} \otimes \cdots \otimes \mathbf{c}_{A_{n-1}} \otimes \mathbf{i d}_{! A_{n}} \otimes \mathbf{i d}_{\Gamma}\right) \text {; } \\
& \left(\mathbf{l l}_{! A_{1}}\left(\ldots \mathbf{l l}_{! A_{n-1}}\left(\left(\mathbf{i d} !_{A_{1} \otimes ! A_{1}} \otimes \cdots \otimes \mathbf{i d}_{! A_{n-1} \otimes ! A_{n-1}} \otimes \mathbf{c}_{A_{n}} \otimes \mathbf{i d}_{\Gamma}\right) ; \mathbf{l l}_{! A_{n}}\left(\left(\mathbf{p l}(\sigma) \otimes \mathbf{p l}(\sigma) \otimes \mathbf{i d}_{\Gamma}\right) ; \tau\right)\right)\right)\right) \\
& =\left(\mathbf{c}_{A_{1}} \otimes \mathbf{i d}_{! A_{2}} \otimes \cdots \otimes \mathbf{i d}_{! A_{n-1}} \otimes \mathbf{i d} \mathbf{d}_{\Gamma}\right) \text {; }
\end{aligned}
$$

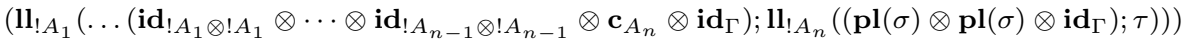


- Promotion-promotion: If $\sigma: A_{1} \otimes \cdots \otimes A_{n} \multimap B$ and $\tau: B \otimes B_{1} \otimes \cdots \otimes B_{m} \vdash C$, we have:

$$
\begin{aligned}
& \left(\mathbf{p l}(\sigma) \otimes \mathbf{i d}_{! B_{1} \otimes \cdots \otimes ! B_{m}}\right) ; \mathbf{p l}(\tau)=\mathbf{p}\left(\left(\mathbf{l}(\sigma) \otimes \mathbf{i d}_{\downarrow B_{1} \otimes \cdots \otimes \downarrow B_{m}}\right) ; \mathbf{l}(\tau)\right) \\
& =\mathbf{p}\left(\mathbf{l l}_{A_{1}}\left(\ldots \mathbf{l l}_{A_{n}}\left(\left(\mathbf{r l}(\sigma) \otimes \mathbf{i d} \mathbf{d}_{\downarrow B_{1} \otimes \cdots \otimes \downarrow B_{m}}\right) ; \mathbf{l}(\tau)\right)\right)\right) \\
& =\mathbf{p}\left(\mathbf{l l}_{A_{1}}\left(\ldots \mathbf{l}_{A_{n}}\left(\left(\mathbf{r l}(\sigma) \otimes \mathbf{i d}_{\downarrow B_{1} \otimes \cdots \otimes \downarrow B_{m}}\right) ; \mathbf{l l}_{B}\left(\mathbf{l l}_{B_{1}}\left(\ldots \mathbf{l l}_{B_{m}}(\mathbf{r l}(\tau))\right)\right)\right)\right)\right) \\
& =\mathbf{p}\left(\mathbf{l l}_{A_{1}}\left(\ldots \mathbf{l}_{A_{n}}\left(\left(\sigma \otimes \mathbf{i d}_{\downarrow B_{1} \otimes \cdots \otimes \downarrow B_{m}}\right) ; \mathbf{l l}_{B_{1}}\left(\ldots \mathbf{l}_{B_{m}}(\mathbf{r l}(\tau))\right)\right)\right)\right) \\
& =\mathbf{p}\left(\mathbf{l l}_{A_{1}}\left(\ldots \mathbf{l l}_{A_{n}}\left(\mathbf{l l}_{B_{1}}\left(\ldots \mathbf{l}_{B_{m}}\left(\left(\sigma \otimes \mathbf{i d}_{B_{1} \otimes \cdots \otimes B_{m}}\right) ; \mathbf{r l}(\tau)\right)\right)\right)\right)\right) \\
& =\mathbf{p}\left(\mathbf{l l}_{A_{1}}\left(\ldots \mathbf{l l}_{A_{n}}\left(\mathbf{l l}_{B_{1}}\left(\ldots \mathbf{l}_{B_{m}}\left(\mathbf{r l}\left(\left(\sigma \otimes \mathbf{i d}_{B_{1} \otimes \cdots \otimes B_{m}}\right) ; \tau\right)\right)\right)\right)\right)\right) \\
& =\mathbf{p l}\left(\left(\sigma \otimes \mathbf{i d}_{B_{1} \otimes \cdots \otimes B_{m}}\right) ; \tau\right)
\end{aligned}
$$

We extend the interpretation to the formula $\mathbb{U}$ and to pseudo-rules. The pseudo-rule $a$ is interpreted by the strategy $\left\{\varepsilon, X^{\mathrm{O}} \cdot X^{\mathrm{P}}\right\}$. If $\sigma$ is the interpretation of the premise of an application of the pseudo-rule $w$, its conclusion is interpreted by $\{\varepsilon\} \cup\left\{m \cdot X^{\mathrm{O}} \mid \exists m s \in \sigma\right\} \cup\left\{m \cdot X^{\mathrm{O}} \cdot X^{\mathrm{P}} \cdot s \mid m s \in\right.$ $\sigma\} . X^{\mathrm{O}}$ denotes $\alpha^{\mathrm{O}}$ if $X=\alpha$ and $\mathrm{q}$ if $X=\mathbb{U}$. $X^{\mathrm{P}}$ denotes $\alpha^{\mathrm{P}}$ if $X=\alpha$ and a if $X=\mathbb{U}$.

If $\sigma$ is the interpretation of a (pseudo)-proof, then $\sigma$ is total.

\section{Realizability}

In order to prove properties of the strategies interpreting proofs, we are going to define a notion of realizability between strategies and formulas.

The relations " $\sigma$ P-realizes $A$ ", $\sigma \Vdash^{P} A$, (with $\sigma$ strategy over $A$ ) and " $\tau$ O-realizes $A$ ", $\tau \Vdash^{O} A$, (with $\tau$ strategy over $A \multimap \mathbb{U}$ ) are defined in a mutually recursive way by induction on $A$ :

- $\sigma \Vdash^{P} \alpha$ if $\sigma=\left\{\varepsilon, \alpha^{\mathrm{O}} \cdot \alpha^{\mathrm{P}}\right\}$

- $\tau \Vdash^{O} \alpha$ if $\tau=\left\{\varepsilon, \mathbf{q} \cdot \alpha^{\mathrm{O}}, \mathbf{q} \cdot \alpha^{\mathrm{O}} \cdot \alpha^{\mathrm{P}} \cdot \mathrm{a}\right\}$

- $\sigma \Vdash^{P} \mathbb{U}$ if $\sigma=\{\varepsilon, \mathrm{q} \cdot \mathrm{a}\}$

- $\tau \Vdash^{O} \mathbb{U}$ if $\tau=\mathbf{i d} \mathbb{U}$

- $\sigma \Vdash^{P} A \otimes B$ if $\sigma_{A} \Vdash^{P} A$ and $\sigma_{B} \Vdash^{P} B$ with $\sigma_{A}=\left\{s_{A} \mid s \in \sigma\right\}$. (We ask in particular that $\sigma_{A}$ and $\sigma_{B}$ are strategies over $A$ and $B$, respectively.)

- $\tau \Vdash^{O} A \otimes B$ if for any $\sigma \Vdash^{P} A, \sigma ; \tau \Vdash^{O} B$ and for any $\sigma \Vdash^{P} B, \sigma ; \tau \Vdash^{O} A$. (Using that, up to the curryfication isomorphisms, $\tau$ can also be seen as a strategy over $A \multimap(B \multimap \mathbb{U})$ or over $B \multimap(A \multimap \mathbb{U})$.)

- $\sigma \Vdash^{P} A \multimap B$ if for any $\delta \Vdash^{P} A, \delta ; \sigma \Vdash^{P} B$ and for any $\tau \Vdash^{O} B, \sigma ; \tau \Vdash^{O} A$

- $\tau \Vdash^{O} A \multimap B$ if $\tau_{A} \Vdash^{P} A$ and $\tau_{B \rightarrow \mathbb{U}} \Vdash^{O} B$

- $\sigma \Vdash^{P} ! A$ if for any exponential signature $t,\left.\sigma\right|_{t} \Vdash^{P} A$ with $\sigma \uparrow_{t}=\left\{s \uparrow_{t} \mid s \in \sigma\right\}$ and $s \uparrow_{t}$ is obtained from $s_{t}$ by replacing any move $(t, m)$ by $m$ and by then erasing the initial open and close moves if they appear (we ask in particular that $\sigma \uparrow_{t}$ is a strategy over $A$ for any $t$ ).

- $\tau \Vdash^{O}$ !A if $\tau$ contains the play $\mathrm{q} \cdot(\mathrm{e}$, open) or the play $\mathrm{q} \cdot(\mathrm{f}, \mathrm{open})$.

Lemma 1 If $\sigma_{1} \Vdash^{P} A \multimap B$ and $\sigma_{2} \Vdash^{P} B \multimap C$ then $\sigma_{1} ; \sigma_{2} \Vdash^{P} A \multimap C$.

Proof. If $\delta \Vdash^{P} A$ then $\delta ;\left(\sigma_{1} ; \sigma_{2}\right)=\left(\delta ; \sigma_{1}\right) ; \sigma_{2} \Vdash^{P} C$. If $\tau \Vdash^{O} C$ then $\left(\sigma_{1} ; \sigma_{2}\right) ; \tau=\sigma_{1} ;\left(\sigma_{2} ; \tau\right) \Vdash^{O}$ A.

We now give an adequacy property relating proofs, strategies and realizability.

Proposition 3 For every proof $\pi$, the strategy $\llbracket \pi \rrbracket$-realizes the conclusion of $\pi$.

Proof. A first remark is that if $\sigma \Vdash^{P} A$ then $\sigma$ contains a non-empty play and if $\tau \Vdash^{O} A$ then $\tau$ contains a play with a move in $A$ (by induction on $A$ ). We now do the proof by induction on $\pi$.

- Axiom: $\llbracket \pi \rrbracket=\mathbf{i d}_{A}$ thus if $\delta \Vdash^{P} A$ then $\delta$; $\llbracket \rrbracket=\delta \Vdash^{P} A$ and if $\tau \Vdash^{O} A, \llbracket \pi \rrbracket ; \tau=\tau \Vdash^{O} A$.

- Cut: if $\sigma_{1} \Vdash^{P} \Gamma \multimap A$ and $\sigma_{2} \Vdash^{P} A \multimap(\Delta \multimap B)$, if $\delta \Vdash^{P} \Gamma$, we have $\delta ;\left(\sigma_{1} ; \sigma_{2}\right)=\left(\delta ; \sigma_{1}\right) ; \sigma_{2}$ with $\delta ; \sigma_{1} \Vdash^{P} A$ thus $\delta ;\left(\sigma_{1} ; \sigma_{2}\right) \Vdash^{P} \Delta \multimap B$. If $\tau \Vdash^{O} \Delta \multimap B$, we have $\left(\sigma_{1} ; \sigma_{2}\right) ; \tau=\sigma_{1} ;\left(\sigma_{2} ; \tau\right)$ with $\sigma_{2} ; \tau \Vdash^{O} A$ thus $\left(\sigma_{1} ; \sigma_{2}\right) ; \tau \Vdash^{O} \Gamma$. 
- Right tensor: if $\sigma_{1} \Vdash^{P} \Gamma \multimap A$ and $\sigma_{2} \Vdash^{P} \Delta \multimap B$, and if $\delta \Vdash^{P} \Gamma \otimes \Delta$ then $\delta_{\Gamma} \Vdash^{P} \Gamma$ and $\delta_{\Delta} \Vdash^{P} \Delta$ so that $\delta_{\Gamma} ; \sigma_{1} \Vdash^{P} A$ and $\delta_{\Delta} ; \sigma_{2} \Vdash^{P} B$, and finally $\delta ;\left(\sigma_{1} \otimes \sigma_{2}\right) \Vdash^{P} A \otimes B$. If $\tau \Vdash^{O} A \otimes B$, $\delta_{1} \Vdash^{P} \Gamma$ and $\delta_{2} \Vdash^{P} \Delta$, we have: $\left(\delta_{1} \otimes \mathbf{i d}_{\Delta}\right) ;\left(\sigma_{1} \otimes \sigma_{2}\right) ; \tau=\left(\delta_{1} ; \sigma_{1}\right) \otimes \sigma_{2} ; \tau=\sigma_{2} ;\left(\left(\delta_{1} ; \sigma_{1}\right) ; \tau\right)$, but $\delta_{1} ; \sigma_{1} \Vdash^{P} A$ thus $\left(\delta_{1} ; \sigma_{1}\right) ; \tau \Vdash^{O} B$ and $\left(\delta_{1} \otimes \mathbf{i d}_{\Delta}\right) ;\left(\sigma_{1} \otimes \sigma_{2}\right) ; \tau \Vdash^{O} \Delta$. In a similar way $\left(\mathbf{i d}_{\Gamma} \otimes \delta_{2}\right) ;\left(\sigma_{1} \otimes \sigma_{2}\right) ; \tau \Vdash^{O} \Gamma$.

- Left tensor: the interpretation of the conclusion of the rule is the same as the interpretation of the premise.

- Right implication: If $\sigma \Vdash^{P}(\Gamma \otimes A) \multimap B$ and $\sigma^{\prime}$ is obtained from $\sigma$ by curryfication, assume $\delta_{1} \Vdash^{P} \Gamma$ and $\delta_{2} \Vdash^{P} A$ then $\delta_{1} ;\left(\delta_{2} ; \sigma^{\prime}\right)=\left(\delta_{1} \otimes \delta_{2}\right) ; \sigma \Vdash^{P} B$. If $\tau \Vdash^{O} A \multimap B, \tau_{B} \Vdash^{O} B$ thus $\sigma ; \tau_{B} \Vdash^{O} \Gamma \otimes A$. Since $\tau_{A} \Vdash^{P} A$ we have $\tau_{A} ;\left(\sigma ; \tau_{B}\right) \Vdash^{O} \Gamma$ and finally $\sigma^{\prime} ; \tau \Vdash^{O} \Gamma$.

- Left implication: If $\sigma_{1} \Vdash^{P} \Gamma \multimap A$ and $\sigma_{2} \Vdash^{P}(\Delta \otimes B) \multimap C$ (with $\sigma^{\prime}: \Gamma \otimes \Delta \otimes(A \multimap B) \multimap C$ obtained by decurryfication from $\sigma_{1} \multimap \sigma_{2}^{\prime}$ where $\sigma_{2}^{\prime}: B \multimap(\Delta \multimap C)$ by curryfication of $\left.\sigma_{2}\right)$ and if $\delta \Vdash^{P} \Gamma \otimes \Delta \otimes(A \multimap B)$ then $\delta ; \sigma^{\prime}=\left(\delta_{\Gamma} ; \sigma_{1}\right) ; \delta_{A \multimap B} ;\left(\delta_{\Delta} ; \sigma_{2}^{\prime \prime}\right) \Vdash^{P} C$ (with $\sigma_{2}^{\prime \prime}: \Delta \multimap(B \multimap C)$ by curryfication of $\left.\sigma_{2}\right)$, using $\sigma_{2}^{\prime \prime} \Vdash^{P} \Delta \multimap(B \multimap C)$ coming from the Right implication case. If $\tau \Vdash^{O} C, \delta_{1} \Vdash^{P} \Gamma, \delta_{2} \Vdash^{P} \Delta$ and $\delta_{3} \Vdash^{P} A \multimap B$ then $\left(\delta_{1} \otimes \delta_{2}\right) ;\left(\sigma^{\prime} ; \tau\right)=\left(\delta_{1} ; \sigma_{1}\right) \multimap$ $\left(\delta_{2} ;\left(\sigma_{2} ; \tau\right)\right) \Vdash^{O} A \multimap B$ since $\delta_{1} ; \sigma_{1} \Vdash^{P} A$ and $\delta_{2} ; \sigma_{2} ; \tau \Vdash^{O} B$. Moreover $\left(\delta_{2} \otimes \delta_{3}\right) ;\left(\sigma^{\prime} ; \tau\right)=$ $\sigma_{1} ; \delta_{3} ;\left(\left(\delta_{2} ; \sigma_{2}\right) ; \tau\right) \Vdash^{O} \Delta$ and $\left(\delta_{1} \otimes \delta_{3}\right) ;\left(\sigma^{\prime} ; \tau\right)=\left(\delta_{1} ; \sigma_{1}\right) ; \delta_{3} ;\left(\sigma_{2} ; \tau\right) \Vdash^{O} \Delta$.

- Weakening: if $\sigma \Vdash^{P} \Gamma \multimap B$ (with $\sigma^{\prime}$ obtained from $\sigma$ by interpreting the weakening rule) and if $\delta \Vdash^{P} \Gamma \otimes ! A$ then $\delta_{\Gamma} \Vdash^{P} \Gamma$ and $\delta_{!} \Vdash^{P} ! A$ so that $\left(\delta_{!_{A}}\right)_{\mathrm{f}} \Vdash^{P} A$. Since $\delta_{!_{A}}$ is thus containing the play $(\mathrm{f}$, open $) \cdot(\mathrm{f}$, close $)$, we can deduce $\delta ; \sigma^{\prime}=\left(\delta_{\Gamma} \otimes \delta_{!}\right) ; \sigma^{\prime}=\delta_{\Gamma} ; \sigma \Vdash^{P} B$. If $\tau \Vdash^{O} B$, $\delta_{1} \Vdash^{P} \Gamma$ and $\delta_{2} \Vdash^{P} ! A$ then $\delta_{1} ;\left(\sigma^{\prime} ; \tau\right)$ plays (f, open) as first move in $! A$ since $\tau$ plays in $B$, and $\delta_{2} ;\left(\sigma^{\prime} ; \tau\right)=\sigma ; \tau \Vdash^{O} \Gamma$.

- Contraction: if $\sigma \Vdash^{P} \Gamma \otimes ! A \otimes ! A \multimap B$ (with $\sigma^{\prime}$ obtained from $\sigma$ by interpreting the contraction rule) and if $\delta \Vdash^{P} \Gamma \otimes ! A$ then $\delta_{\Gamma} \Vdash^{P} \Gamma$ and $\delta_{! A} \Vdash^{P} ! A$ so that we can define $\delta^{1} \Vdash^{P} ! A$ and $\delta^{2} \Vdash^{P} ! A$ (by $\delta^{1}=\left\{\left(t_{1}, m_{1}\right) \cdots\left(t_{2 n}, m_{2 n}\right) \mid\left(\mathrm{I}\left(t_{1}\right), m_{1}\right) \cdots\left(\mathrm{I}\left(t_{2 n}\right), m_{2 n}\right) \in \delta\right\}$, and similarly with $\mathrm{r}$ for $\left.\delta^{2}\right)$ with $\delta ; \sigma^{\prime}=\left(\delta_{\Gamma} \otimes \delta^{1} \otimes \delta^{2}\right) ; \sigma \Vdash^{P} B$. If $\tau \Vdash^{O} B, \delta_{1} \Vdash^{P} \Gamma$ and $\delta_{2} \Vdash^{P} ! A$ (allowing us to define, as above, $\delta_{2}^{1} \Vdash^{P} ! A$ and $\left.\delta_{2}^{2} \Perp^{P} ! A\right)$ then $\delta_{1} ;\left(\sigma^{\prime} ; \tau\right)$ plays (f, open) as first move in $! A$ since $\tau$ plays in $B$, and $\delta_{2} ;\left(\sigma^{\prime} ; \tau\right)=\left(\delta_{2}^{1} \otimes \delta_{2}^{2}\right) ; \sigma ; \tau \Vdash^{O} \Gamma$.

- Dereliction: if $\sigma \Vdash^{P} \Gamma \otimes A \multimap B$ (with $\sigma^{\prime}$ obtained from $\sigma$ by interpreting the dereliction rule) and if $\delta \Vdash^{P} \Gamma \otimes ! A$ then $\delta_{\Gamma} \Vdash^{P} \Gamma$ and $\delta_{! A} \Vdash^{P} ! A$ so that $\left(\delta_{! A}\right)_{\mathrm{e}} \Vdash^{P} A$ and $\delta ; \sigma^{\prime}=$ $\left(\delta_{\Gamma} \otimes\left(\delta_{!}\right)_{\mathrm{e}}\right) ; \sigma \Vdash^{P} B$. If $\tau \Vdash^{O} B, \delta_{1} \Vdash^{P} \Gamma$ and $\delta_{2} \Vdash^{P} ! A$ then $\delta_{1} ;\left(\sigma^{\prime} ; \tau\right)$ plays (e, open) as first move in ! $A$ since $\tau$ plays in $B$, and $\delta_{2} ;\left(\sigma^{\prime} ; \tau\right)=\left(\delta_{2}\right) \Gamma_{\mathrm{e}} ; \sigma ; \tau \Vdash^{O} \Gamma$.

- Digging: if $\sigma \Vdash^{P} \Gamma \otimes ! ! A \multimap B$ (with $\sigma^{\prime}$ obtained from $\sigma$ by interpreting the digging rule) and if $\delta \Vdash^{P} \Gamma \otimes ! A$ then $\delta_{\Gamma} \Vdash^{P} \Gamma$ and $\delta_{!} \Vdash^{P} ! A$ so that we can define $\delta^{\prime} \Vdash^{P} ! ! A$ (by $\left.\delta^{\prime}=\left\{\left(t_{1},\left(s_{1}, m_{1}\right)\right) \cdots\left(t_{2 n},\left(s_{2 n}, m_{2 n}\right)\right) \mid\left(\mathrm{n}\left(t_{1}, s_{1}\right), m_{1}\right) \cdots\left(\mathrm{n}\left(t_{2 n}, s_{2 n}\right), m_{2 n}\right) \in \delta\right\}\right)$ with $\delta ; \sigma^{\prime}=$ $\left(\delta_{\Gamma} \otimes \delta^{\prime}\right) ; \sigma \Vdash^{P} B$. If $\tau \Vdash^{O} B, \delta_{1} \Vdash^{P} \Gamma$ and $\delta_{2} \Vdash^{P} ! A$ (allowing us to define, as above, $\delta_{2}^{\prime} \Vdash^{P} ! ! A$ ) then $\delta_{1} ;\left(\sigma^{\prime} ; \tau\right)$ plays (f, open) as first move in $! A$ since $\tau$ plays in $B$, and $\delta_{2} ;\left(\sigma^{\prime} ; \tau\right)=\delta_{2}^{\prime} ; \sigma ; \tau \Vdash O$ $\Gamma$.

- Promotion: if $\sigma \Vdash^{P} A_{1} \otimes \cdots \otimes A_{n} \multimap B$ (with $\sigma^{\prime}$ obtained from $\sigma$ by interpreting the promotion rule) and if $\delta_{i} \Vdash^{P} ! A_{i}(1 \leq i \leq n)$, for any exponential signature $t$ we have $\delta_{i} \uparrow_{t} \Vdash^{P} A_{i}$ thus $\left(\left(\delta_{1} \otimes \cdots \otimes \delta_{n}\right) ; \sigma^{\prime}\right) \uparrow_{t}=\left(\delta_{1} \uparrow_{t} \otimes \cdots \otimes \delta_{n} \uparrow_{t}\right) ; \sigma \Vdash^{P} B$. If $\tau \Vdash^{O} ! B$ and $\delta_{i} \Vdash^{P} ! A_{i}(1 \leq i \leq n)$, for any $1 \leq i \leq n,\left(\delta_{1} \otimes \cdots \otimes \delta_{i-1} \otimes \mathbf{i d}_{!_{i}} \otimes \delta_{i+1} \otimes \cdots \otimes \delta_{n}\right) ; \sigma^{\prime} ; \tau$ plays $(t$, open) with $t=\mathrm{e}$ or $t=\mathrm{f}$ as first move in ! $A_{i}$ since $\tau$ plays such a $\left(t\right.$, open) as first move in $! B$ and each $\delta_{i}$ contains the play $(t$, open $) \cdot(t$, close $)$.

- Pseudo-rule $a: \sigma \Vdash^{P} X$ by definition.

- Pseudo rule $w$ : if $\sigma \Vdash^{P} \Gamma \multimap B$ (with $\sigma^{\prime}$ obtained from $\sigma$ by interpreting the $w$ pseudo-rule) and if $\delta \Vdash^{P} \Gamma \otimes X$ then $\delta_{\Gamma} \Vdash^{P} \Gamma$ and $\delta_{X} \Vdash^{P} X$. Since $\delta_{X}$ is thus containing the play $X^{\mathrm{O}} \cdot X^{\mathrm{P}}$, we can deduce $\delta ; \sigma^{\prime}=\left(\delta_{\Gamma} \otimes \delta_{X}\right) ; \sigma^{\prime}=\delta_{\Gamma} ; \sigma \Vdash^{P} B$. If $\tau \Vdash^{O} B, \delta_{1} \Vdash^{P} \Gamma$ and $\delta_{2} \Vdash^{P} X$ then $\delta_{1} ;\left(\sigma^{\prime} ; \tau\right)$ contains $X^{\mathrm{O}} \cdot X^{\mathrm{P}}$ and $\delta_{2} ;\left(\sigma^{\prime} ; \tau\right)=\sigma ; \tau \Vdash^{O} \Gamma$.

As a consequence, $\llbracket \mathrm{TA} \mathrm{A}_{A} \rrbracket \Vdash^{P} A \multimap \mathbb{U}$ thus $\llbracket \mathrm{TA}_{A} \rrbracket \Perp^{O} A$ (since $\mathbf{i d}_{\mathbb{U}} \Vdash^{O} \mathbb{U}$ ).

A complete set of moves for any game $A$ is a subset of $M_{A}$ defined by induction on the structure of $A$ : 
- If $A=\alpha$, the only complete set of moves for $A$ is $\left\{\alpha^{\mathrm{P}}, \alpha^{\mathrm{O}}\right\}$.

- If $A=B \otimes C$ or $A=B \multimap C, C_{B}$ is a complete set of moves for $B$ and $C_{C}$ is a complete set of moves for $C$, then $C_{A}=C_{B}+C_{C}$ is a complete set of moves for $A$.

- If $A=! B$, then any subset of $M_{A}$ containing the move (e, close) or the move ( $\mathrm{f}$, close) is a complete set of move for $A$.

Proposition 4 If $\sigma$ P-realizes $A, \tau$ O-realizes $A$ and $\sigma ; \tau$ is total, then the maximal sequence in $\sigma \| \tau$ (seen as a set of moves of $A$ ) is complete.

Proof. By induction on $A$ :

- If $A=\alpha$, then $\sigma=\left\{\varepsilon, \alpha^{\mathrm{O}} \cdot \alpha^{\mathrm{P}}\right\}$ and $\tau=\left\{\varepsilon, \mathbf{q} \cdot \alpha^{\mathrm{O}}, \mathbf{q} \cdot \alpha^{\mathrm{O}} \cdot \alpha^{\mathrm{P}} \cdot \mathrm{a}\right\}$. As a consequence, $\sigma \| \tau$ is a complete set of moves.

- If $A=B \otimes C$, then

$$
\sigma\left\|\tau=\left(\sigma_{B} \otimes \sigma_{C}\right)\right\| \tau \supseteq \sigma_{B} \| \sigma_{C} ; \tau .
$$

But both $\sigma_{C} ; \tau \Vdash^{O} B$ and $\sigma_{B} \Vdash^{P} B$. By IH, $\sigma \| \tau$ contains a complete set of moves for $B$. Symmetrically:

$$
\sigma\left\|\tau=\left(\sigma_{B} \otimes \sigma_{C}\right)\right\| \tau \supseteq \sigma_{C} \| \sigma_{B} ; \tau .
$$

Moreover, $\sigma_{B} ; \tau \Vdash^{O} C$ and $\sigma_{C} \Vdash^{P} C$. By IH, $\sigma \| \tau$ contains a complete set of moves for $C$.

- If $A=B \multimap C$, then

$$
\sigma\left\|\tau \supseteq \tau_{B} ; \sigma\right\| \tau_{C \multimap \mathbb{U}} .
$$

But $\tau_{B} \Vdash^{P} B$, which implies $\tau_{B} ; \sigma \Vdash^{P} C$. Moreover, $\tau_{C \rightarrow \mathbb{U}} \Vdash^{O} C$ and, by IH, $\sigma \| \tau$ contains a complete set of moves for $C$. Symmetrically:

$$
\sigma\left\|\tau \supseteq \tau_{B}\right\| \sigma ; \tau_{C-\mathbb{U}}
$$

from which it follows that $\sigma \| \tau$ contains a complete set of moves for $B$.

- If $A=! B, \sigma ; \tau \neq\{\varepsilon\}$ entails that $\sigma \| \tau$ is not empty thus contains the move ( $t$,open) (with $t=\mathrm{e}$ or $t=\mathrm{f}$ ) played by (player in) $\tau$ in $A$. Then it also contains the move ( $t$, close) played by (player in) $\sigma$ and it is a complete set of moves. Notice we haven't used the IH here.

This concludes the proof.

\section{Complexity}

In this Section, we show how to instrument games with slots, in the same vein as in Ghica's framework [7]. The idea is simple: slots are used by the player to communicate some quantitative properties of the underlying proof to the opponent. But while in Ghica's work slots are produced in correspondence with any potential redex, here the player raises a slot in correspondence with boxes, i.e. instances of the promotion rule. In Ghica's slot games, the complexity of a program can be read out of any complete play in its interpretation, while here the process of measuring the complexity of proofs is internalized through the notion of time analyzer (see Section 2): the complexity of $\pi$ (with conclusion $A$ ) is simply the number of slots produced in the interaction between $\llbracket \pi \rrbracket$ and $\llbracket \mathrm{TA}_{A} \rrbracket$. Notice that the definition of $\mathrm{TA}_{A}$ only depends on the formula $A$.

The symbol $\bullet$ is a special symbol called a slot. In the new setting, the set of moves for $A$, will be the usual $M_{A}$, while the notion of a play should be slightly changed. Given a game $A$ and a sequence $s$ in $\left(M_{A} \cup\{\bullet\}\right)^{*}$, we denote by $s^{\circ}$ the sequence in $M_{A}^{*}$ obtained by deleting any occurrence of $\bullet$ in $s$. Analogously, given any subset $\sigma$ of $\left(M_{A} \cup\{\bullet\}\right)^{*}, \sigma^{\circ}$ will denote $\left\{s^{\circ} \mid s \in \sigma\right\} \subseteq M_{A}^{*}$.

A play-with-costs for $A$ is a sequence $s$ in $\left(M_{A} \cup\{\bullet\}\right)^{*}$ such that $s^{\circ} \in P_{A}$, whenever $s=r \bullet m q$ it holds that $\lambda_{A}(m)=\mathrm{P}$ and the last symbol in $s$ (if any) is a move in $M_{A}$. A strategy-with-costs for the game $A$ is a set $\sigma$ of plays-with-costs for $A$ such that $\sigma^{\circ}$ is a strategy (in the usual sense) for $A$ and, moreover, $\sigma$ is slot-deterministic: if $s m \bullet^{k} n \in \sigma$ and $s m \bullet^{h} n \in \sigma$, then $k=h$.

Composition of strategies-with-costs needs to be defined in a slightly different way than the one of usual strategies. In particular, we need two different notions of projections: first of all, if $s \in\left(M_{A} \cup M_{B} \cup\{\bullet\}\right)^{*}$, we can construct $s_{A^{X}}$ (where $X \subseteq\{\mathrm{P}, \mathrm{O}\}$ ) by extracting from $s$ any move 
$m \in M_{A}$ together with the slots immediately before any such $m$ provided $\lambda_{A}(m) \in X$. But we can even construct $s_{A} \bullet$, by only considering the slots which precede moves in $M_{A}$ but not the moves themselves. Given strategies-with-costs $\sigma$ over $A \multimap B$ and $\tau$ over $B \multimap C$, we can first define $\sigma \| \tau$ as follows:

$$
\sigma \| \tau=\left\{s \in\left(M_{A} \cup M_{B} \cup M_{C} \cup\{\bullet\}\right)^{*} \mid s_{A^{\mathrm{P}, \mathrm{o}}, B^{\mathrm{P}}} \in \sigma \wedge s_{B^{\mathrm{O}}, C^{\mathrm{P}, \mathrm{o}}} \in \tau\right\} .
$$

The composition of $\sigma$ and $\tau$, denoted $\sigma ; \tau$ is now simply

$$
\sigma ; \tau=\left\{s_{A^{\mathrm{P}, \mathrm{O}, B} \bullet, C^{\mathrm{P}, \mathrm{O}}} \mid s \in \sigma \| \tau\right\} .
$$

In other words, we forget the moves in $M_{B}$, but we keep all the slots produced by them.

Proposition 5 If $\sigma$ is a strategy-with-costs over $A \multimap B$ and $\tau$ is a strategy-with-costs over $B \multimap C$, then $\sigma ; \tau$ is a strategy-with-costs over $A \multimap C$.

The strategy constructions we have seen so far can be turned into strategy-with-costs constructions. In the basic strategies, slots come into play only in $\operatorname{rl}(\sigma)$ : in particular, $\operatorname{rl}^{i}(\sigma)=$ $\{\varepsilon\} \cup\left\{\right.$ open $\left._{\downarrow A} \cdot \bullet^{i} \cdot \operatorname{close}_{\downarrow A} \cdot s \mid s \in \sigma\right\}$. This way, the interpretation $\llbracket \pi \rrbracket^{i}$ of any proof $\pi$ is parametrized on a natural number $i$.

We are in a position to define the complexity $\mathcal{C}(\pi)$ of any proof $\pi$. First, consider the shape of any non-trivial play-with-costs $s$ in a strategy-with-costs $\sigma$ for $\mathbb{U}$ : it must have the following shape $\mathrm{q} \bullet^{i}$ a. But observe that this play is the only non-trivial play-with-costs in $\sigma$, due to (slot) determinacy. The integer $i$ is called the complexity of $\sigma$, denoted $\mathcal{C}(\sigma)$. This way we can define the complexity $\mathcal{C}(\pi)$ of any proof $\pi$ with conclusion $A$ as simply the complexity of $\pi$ when composed with the time analyzer: $\mathcal{C}\left(\llbracket \pi \rrbracket^{1} ; \llbracket T A_{A} \rrbracket^{0}\right)$. The complexity of $\pi$ is defined for every $\pi$ because $\llbracket \pi \rrbracket ; \llbracket \mathrm{TA}_{A} \rrbracket P$-realizes $\mathbb{U}$ (by Proposition 3 ) and, as a consequence, contains a non-empty play. Given any play-with-costs $s, \mathcal{C}(s)$ is simply the number of occurrences of $\bullet$ in $s$.

\subsection{Dynamics Under Exposed Cuts}

In this Section, we will prove some lemmas about the preservation of semantics when cuts are exposed as in the [.] construction (see Section 2). With $\llbracket \pi \rrbracket_{e c a}$ we denote the (unique) maximal (wrt the prefix order) sequence in $\iota \|\left(\llbracket \pi \rrbracket_{e c}^{1} ; \llbracket^{T} \mathrm{TA}_{B} \rrbracket^{0}\right)$, where $[\pi]: !^{k_{1}}\left(A_{1} \multimap A_{1}\right), \ldots, !^{k_{n}}\left(A_{n} \multimap\right.$ $\left.A_{n}\right), \Gamma \vdash C, \llbracket \pi \rrbracket_{e c}^{i}=\llbracket\left[\pi \rrbracket \rrbracket^{i}, \iota=\llbracket !^{k_{1}} \mathbf{i d}_{A_{1}} \otimes \cdots \otimes !^{k_{n}} \mathbf{i d}_{A_{n}} \rrbracket^{0}\right.$ and $B=\otimes \Gamma \multimap C . !^{k} \mathbf{i d}_{A}$ is the strategy over $!^{k}(A \multimap A)$ obtained by applying $k$ times the interpretation of the promotion rule (with an empty context) to the identity strategy. We are interested in studying how $\llbracket \pi \rrbracket_{\text {eca }}$ evolves during cut elimination for any proof $\pi: \Gamma \vdash C$. This will lead us to full abstraction. Indeed:

Remark 1 Please notice that the strategy from which we obtain the complexity of $\pi$ is

$$
\tau=\llbracket \pi \rrbracket^{1} ; \llbracket T A_{B} \rrbracket^{0}=\left(\iota ; \llbracket \pi \rrbracket_{e c}^{1}\right) ; \llbracket T A_{B} \rrbracket^{0}=\iota ;\left(\llbracket \pi \rrbracket_{e c}^{1} ; \llbracket T A_{B} \rrbracket^{0}\right) .
$$

This implies that $\llbracket \pi \rrbracket_{\text {eca }}$ contains exactly $\mathcal{C}(\pi)$ slots and, moreover, it contains a complete set of moves for $D=!^{k_{1}}\left(A_{1} \multimap A_{1}\right) \otimes \cdots \otimes !^{k_{n}}\left(A_{n} \multimap A_{n}\right)$. This, in particular, is a consequence of Proposition 4 , since $\iota \Vdash^{P} D,\left(\llbracket \pi \rrbracket_{e c}^{1} ; \llbracket T A_{B} \rrbracket^{0}\right) \Vdash^{O} D$ and their composition is total.

The cut-elimination relation $\rightsquigarrow$ can be thought of as the union of nine reduction relations $\stackrel{x}{\rightsquigarrow}$ where $x$ ranges over the set $\mathcal{R}=\{\mathcal{T}, \mathcal{X}, \otimes, \multimap, \mathcal{C}, \mathcal{D}, \mathcal{N}, \mathcal{W}, !-!\}$. They correspond to commuting, axiom, tensor, linear arrow, contraction, dereliction, digging, weakening and promotion-promotion cut-elimination steps. If $X \subseteq \mathcal{R}$ or $x \in \mathcal{R}$, then $\stackrel{X}{\rightsquigarrow}$ and $\stackrel{x}{\rightsquigarrow}$ have the obvious meaning. We can consider a reduction relation that postpones !- !-cuts at the very end of the computation. The resulting reduction relation is denoted with $\hookrightarrow$. Again, $\stackrel{X}{\hookrightarrow}$ and $\stackrel{x}{\hookrightarrow}$ (where $x \in \mathcal{R}$ and $X \subseteq \mathcal{R}$ ) have their natural meaning.

We need to analyze how $\llbracket \rho \rrbracket_{e c a}$ differs from $\llbracket \pi \rrbracket_{e c a}$ if $\pi \stackrel{x}{\rightsquigarrow} \rho$. Clearly, this crucially depends on $x \in \mathcal{R}$, since cuts are exposed in $[\pi]$ and $[\rho]$. 
Lemma 2 (Commuting steps) If $\pi \stackrel{\mathcal{T}}{\rightsquigarrow} \rho$, then $\llbracket \pi \rrbracket_{e c a}=\llbracket \rho \rrbracket_{e c a}$.

Proof. Trivial.

Lemma 3 (Axiom) If $\pi \stackrel{\mathcal{X}}{\rightsquigarrow}$, then $\mathcal{C}\left(\llbracket \pi \rrbracket_{\text {eca }}\right)=\mathcal{C}\left(\llbracket \rho \rrbracket_{\text {eca }}\right)$.

Proof. We only consider the case where the cut reduced in $\pi$ is the last rule of $\pi$. The other cases can be reduced to this one by an easy induction. Moreover, we assume that the right premise of the cut under consideration is the conclusion of an axiom (the other case is similar). $\pi$ is the proof

$$
\frac{\sigma_{1}: \Delta_{1} \vdash B_{1} \ldots \sigma_{n}: \Delta_{n} \vdash B_{n}}{\frac{\vartheta(\Gamma) \vdash A}{(\Gamma) \vdash A}} \frac{}{A \vdash A} U
$$

and $\rho$ is simply

$$
\frac{\sigma_{1}: \Delta_{1} \vdash B_{1} \quad \ldots \quad \sigma_{n}: \Delta_{n} \vdash B_{n}}{\varsigma(\Gamma) \vdash A}
$$

Moreover, $[\pi]$ is

$$
\frac{\left[\sigma_{1}\right]: \Psi_{1}, \Delta_{1} \vdash B_{1} \quad \ldots \quad\left[\sigma_{n}\right]: \Psi_{n}, \Delta_{n} \vdash B_{n}}{\frac{\Phi, \vartheta(\Gamma) \vdash A}{A \vdash A}} \frac{}{\Phi,[A \multimap A], \varsigma(\Gamma) \vdash A} L_{\multimap}
$$

and $[\rho]$ is

$$
\frac{\left[\sigma_{1}\right]: \Psi_{1}, \Delta_{1} \vdash B_{1} \quad \ldots \quad\left[\sigma_{n}\right]: \Psi_{n}, \Delta_{n} \vdash B_{n}}{\Phi, \varsigma(\Gamma) \vdash A}
$$

Observe that:

$$
\begin{aligned}
& {[\pi] \quad: \quad \Phi_{1},\left[A_{1} \multimap A_{2}\right]_{x-A}, \Gamma_{1} \vdash A} \\
& {[\rho] \quad: \quad \Phi_{1}, \Gamma_{1} \vdash A}
\end{aligned}
$$

Now, consider the maximal sequence $\llbracket \rho \rrbracket_{e c a}$ in $\iota \|\left(\llbracket \rho \rrbracket_{e c}^{1} ; \llbracket \mathrm{TA}_{D} \rrbracket^{0}\right)$ where $D$ is the conclusion of $\pi$, and the maximal sequence $\llbracket \pi \rrbracket_{e c a}$ in $\iota \|\left(\llbracket \pi \rrbracket_{e c}^{1} ; \llbracket \mathrm{TA}_{D} \rrbracket^{0}\right)$. The following equation holds:

$$
\llbracket \rho \rrbracket_{e c a}=\llbracket \pi \rrbracket_{e c a}\left\{\cdot / m_{A_{1}} m_{A_{2}}, \cdot / m_{A_{2}} m_{A_{1}}\right\}
$$

Notice, however, that any slot in $\llbracket \pi \rrbracket_{e c a}$ is preserved. This includes, in particular, slots coming from any of the $\sigma_{i}$.

Lemma 4 (Tensor) If $\pi \stackrel{\otimes}{\rightsquigarrow} \rho$, then $\llbracket \pi \rrbracket_{e c a}=\llbracket \rho \rrbracket_{e c a}$.

Proof. We only consider the case where the cut reduced in $\pi$ is the last rule of $\pi$. The other cases can be reduced to this one by an easy induction. With this hypothesis, $\pi$ is the proof

$$
\frac{\sigma_{1}: \Omega_{1} \vdash B \quad \sigma_{2}: \Omega_{2} \vdash C}{\frac{\varpi\left(\Omega_{1}, \Omega_{2}\right) \vdash B \otimes C}{\Gamma \vdash A} \frac{\theta: \Delta, B, C \vdash A}{\Delta, B \otimes C \vdash A}} U
$$

and $\rho$ is simply

$$
\frac{\sigma_{1}: \Omega_{1} \vdash B \frac{\sigma_{2}: \Omega_{2} \vdash C \quad \theta: \Delta, B, C \vdash A}{\Omega_{2}, \Delta, B \vdash A}}{\Gamma \vdash A} U
$$

Moreover, $[\pi]$ is

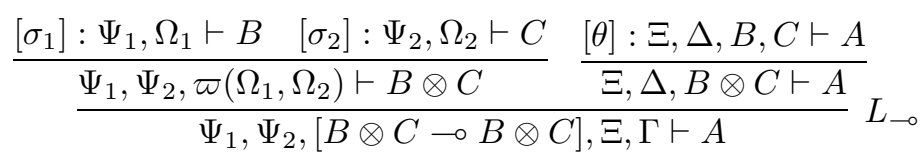


and $[\rho]$ is

$$
\frac{\left[\sigma_{1}\right]: \Psi_{1}, \Omega_{1} \vdash B \quad \frac{\left[\sigma_{2}\right]: \Psi_{2}, \Omega_{2} \vdash C \quad[\theta]: \Xi, \Delta, B, C \vdash A}{\Psi_{2},[C \multimap C], \Xi, \Omega_{2}, \Delta, B \vdash A}}{\Psi_{1},[B \multimap B], \Psi_{2},[C \multimap C], \Xi, \Gamma \vdash A} L_{\multimap}
$$

Now, consider $\llbracket \rho \rrbracket_{e c a}$. We can clearly find the same sequence in $\iota^{\prime} \|\left(\llbracket \pi \rrbracket_{e c}^{1} ; \llbracket \mathrm{TA}_{D} \rrbracket^{0}\right)$.

Lemma 5 (Linear Arrow) If $\pi \stackrel{\circ}{\rightsquigarrow} \rho$, then $\llbracket \pi \rrbracket_{e c a}=\llbracket \rho \rrbracket_{e c a}$.

Proof. We only consider the case where the cut reduced in $\pi$ is the last rule of $\pi$. The other cases can be reduced to this one by an easy induction. With this hypothesis, $\pi$ is the proof

$$
\frac{\frac{\theta: \Delta, B \vdash C}{\Delta \vdash B \multimap C} \frac{\sigma_{1}: \Omega_{1} \vdash B \quad \sigma_{2}, \Omega_{2}, C \vdash A}{\varpi\left(\Omega_{1}, \Omega_{2}\right), B \multimap C \vdash A}}{\Gamma \vdash A} U
$$

and $\rho$ is simply

$$
\frac{\sigma_{1}: \Omega_{1} \vdash B \frac{\theta: \Delta, B \vdash C \quad \sigma_{2}: \Omega_{2}, C \vdash A}{\Delta, \Omega_{2}, B \vdash A}}{\Gamma \vdash A} U
$$

Moreover, $[\pi]$ is

$$
\frac{\frac{\theta: \Psi, \Delta, B \vdash C}{\Psi, \Delta \vdash B \multimap C} \frac{\sigma_{1}: \Xi_{1}, \Omega_{1} \vdash B \quad \sigma_{2}: \Xi_{2}, \Omega_{2}, C \vdash A}{\Xi_{1}, \Xi_{2}, \varpi\left(\Omega_{1}, \Omega_{2}\right), B \multimap C \vdash A}}{\Psi,[(B \multimap C) \multimap(B \multimap C)], \Xi_{1}, \Xi_{2}, \Gamma \vdash A} L_{\multimap}
$$

and $[\rho]$ is

$$
\frac{\sigma_{1}: \Xi_{1}, \Omega_{1} \vdash B \quad \frac{\theta: \Psi, \Delta, B \vdash C \quad \sigma_{2}: \Xi_{2}, \Omega_{2}, C \vdash A}{\Psi,[C \multimap C], \Xi_{2}, \Delta, \Omega_{2}, B \vdash A}}{\Xi_{1},[B \multimap B], \Psi,[C \multimap C], \Xi_{2}, \Gamma \vdash A} L_{\multimap}
$$

Now, consider $\llbracket \rho \rrbracket_{e c a}$. We can clearly find the same sequence in $\iota^{\prime} \|\left(\llbracket \pi \rrbracket_{e c}^{1} ; \llbracket \mathrm{TA}_{D} \rrbracket^{0}\right)$, which is clearly maximal.

Lemma 6 (Dereliction) If $\pi \stackrel{\mathcal{D}}{\rightsquigarrow} \rho$, then $\mathcal{C}\left(\llbracket \pi \rrbracket_{e c a}\right)=\mathcal{C}\left(\llbracket \rho \rrbracket_{e c a}\right)+1$.

Proof. We only consider the case where the cut reduced in $\pi$ is the last rule in $\pi$. The other cases can be reduced to this one by an easy induction. With this hypothesis, $\pi$ is

$$
\frac{\frac{\sigma: D_{1}, \ldots, D_{m} \vdash B}{\varsigma\left(! D_{1}, \ldots, ! D_{m}\right) \vdash ! B} P_{!} \frac{\theta: \Gamma, B \vdash C}{\vartheta(\Gamma), ! B \vdash C}}{\varpi\left(! D_{1}, \ldots, ! D_{m}, \Gamma\right) \vdash C} D !
$$

and $\rho$ is

$$
\begin{aligned}
& \frac{\sigma: D_{1}, \ldots, D_{m} \vdash B \quad \theta: \Gamma, B \vdash C}{\Gamma, D_{1}, \ldots, D_{m} \vdash C} U \\
& \stackrel{\frac{D_{m}, \Gamma, D_{1}, \ldots, D_{m-1} \vdash C}{D_{!}}}{D_{!}} \\
& \frac{\vdots}{\frac{! D_{2}, \ldots, ! D_{m}, \Gamma, D_{1} \vdash C}{\varpi\left(! D_{1}, \ldots, ! D_{m}, \Gamma\right) \vdash C}} D_{!} D_{!}
\end{aligned}
$$

Moreover, $[\pi]$ is

$$
\frac{[\sigma]:\left[A_{1}\right], \ldots,\left[A_{n}\right], D_{1}, \ldots, D_{m} \vdash B}{\left[! A_{1}\right], \ldots,\left[! A_{n}\right], \varsigma\left(! D_{1}, \ldots, ! D_{m}\right) \vdash ! B} P_{!} \frac{[\theta]: \Phi, \Gamma, B \vdash C}{\Phi, \vartheta(\Gamma), ! B \vdash C} D_{!}
$$


and $[\rho]$ is

$$
\begin{aligned}
& \frac{[\sigma]:\left[A_{1}\right], \ldots,\left[A_{n}\right], D_{1}, \ldots, D_{m} \vdash B \quad[\theta]: \Phi, \Gamma, B \vdash C}{\left[A_{1}\right], \ldots,\left[A_{n}\right],[B \multimap B], \Phi, \Gamma, D_{1}, \ldots, D_{m} \vdash C} L_{-} \\
& \frac{\left[A_{1}\right], \ldots,\left[A_{n}\right],[B \multimap B], \Phi, ! D_{m}, \Gamma, D_{1}, \ldots, D_{m-1} \vdash C}{\vdots} D_{!} \\
& \frac{\vdots}{\left[A_{1}\right], \ldots,\left[A_{n}\right],[B \multimap B], \Phi, ! D_{2}, \ldots, ! D_{m}, \Gamma, D_{1} \vdash C} \\
& D_{!} \\
& {\left[A_{1}\right], \ldots,\left[A_{n}\right],[B \multimap B], \Phi, \varpi\left(! D_{1}, \ldots, ! D_{m}, \Gamma\right) \vdash C}
\end{aligned}
$$

Observe that:

$$
\begin{array}{ll}
{[\pi] \quad:} & {\left[! A_{1}\right], \ldots,\left[! A_{n}\right],\left[! B_{1} \multimap ! B_{2}\right]_{P_{!}-D_{!}}, \Phi_{1}, \Gamma_{1} \vdash C} \\
{[\rho]:} & :\left[A_{1}\right], \ldots,\left[A_{n}\right],\left[B_{1} \multimap B_{2}\right], \Phi_{1}, \Gamma_{1} \vdash C
\end{array}
$$

Now, consider $\iota \|\left(\llbracket \rho \rrbracket_{e c}^{1} ; \llbracket \mathrm{TA}_{E} \rrbracket\right)$ and $\iota^{\prime} \|\left(\llbracket \pi \rrbracket_{e c}^{1} ; \llbracket \mathrm{TA}_{E} \rrbracket\right)$, where $E$ is the conclusion of $\rho$ and $\pi$. It is easy to realize that $\llbracket \rho \rrbracket_{e c a}$ can be simulated by the $\llbracket \pi \rrbracket_{e c a}$, in such a way that

$$
\llbracket \rho \rrbracket_{e c a}=\llbracket \pi \rrbracket_{e c a}\left\{m_{B_{i}} /\left(\mathrm{e}, m_{B_{i}}\right), \cdot / \bullet^{a}(\mathrm{e}, \mu)_{! B_{i}}, m_{A_{i}} /\left(\mathrm{e}, m_{A_{i}}\right), \cdot / \bullet(\mathrm{e}, \mu) ! A_{i}\right\}
$$

where $\mu$ is a metavariable for either open or close. Observe that $a=1$ when $\mu=$ close and $i=1$ (a promotion in $\pi$ raises a slot), $a=0$ otherwise and $b=0$ ( $\iota$ does not raise any slot). But there is exactly one (e, close)! $B_{1}$ in $\llbracket \pi \rrbracket_{e c a}$ : at most one for obvious reasons (the same move is not played twice), at least one from Proposition 4 (since strategies interpreting (pseudo)proofs are total). The thesis easily follows.

Lemma 7 (Contraction) If $\pi \stackrel{C}{\rightsquigarrow} \rho$, then $\mathcal{C}\left(\llbracket \pi \rrbracket_{e c a}\right)=\mathcal{C}\left(\llbracket \rho \rrbracket_{e c a}\right)+1$.

Proof. We only consider the case where the cut reduced in $\pi$ is the last rule of $\pi$. The other cases can be reduced to this one by an easy induction. With this hypothesis, $\pi$ is

$$
\frac{\frac{\sigma: D_{1}, \ldots, D_{m} \vdash B}{\varsigma\left(! D_{1}, \ldots, ! D_{m}\right) \vdash ! B} P_{!} \quad \frac{\theta: \Gamma, ! B, ! B \vdash C}{\vartheta(\Gamma), ! B \vdash C}}{\varpi\left(! D_{1}, \ldots, ! D_{m}, \Gamma\right) \vdash C} U
$$

and $\rho$ is

$$
\frac{\frac{\sigma: D_{1}, \ldots, D_{m} \vdash B}{\varsigma\left(! D_{1}, \ldots, ! D_{m}\right) \vdash ! B} P_{!} \frac{\frac{D_{1}, \ldots, D_{m} \vdash B}{\varsigma\left(! D_{1}, \ldots, ! D_{m}\right) \vdash ! B} P_{!} \quad \theta: \Gamma, ! B, ! B \vdash C}{\varsigma\left(! D_{1}, \ldots, ! D_{m}\right), \Gamma, ! B \vdash C}}{\frac{\Gamma, ! D_{1}, ! D_{1}, \ldots, ! D_{m}, ! D_{m} \vdash C}{\frac{! D_{m}, \Gamma, ! D_{1}, ! D_{1}, \ldots, ! D_{m-1}, ! D_{m-1} \vdash C}{\vdots}} C} U
$$

Moreover, $[\pi]$ is

$$
\frac{[\sigma]:\left[A_{1}\right], \ldots,\left[A_{n}\right], D_{1}, \ldots, D_{m} \vdash B}{\left[! A_{1}\right], \ldots,\left[! A_{n}\right], \varsigma\left(! D_{1}, \ldots, ! D_{m}\right) \vdash ! B} P_{!} \frac{[\theta]: \Phi, \Gamma, ! B, ! B \vdash C}{\Phi, \vartheta(\Gamma), ! B \vdash C} C_{-}
$$

and $[\rho]$ is

$$
\begin{aligned}
& \frac{[\sigma:]:\left[A_{1}\right], \ldots,\left[A_{n}\right], D_{1}, \ldots, D_{m} \vdash B}{\left[! A_{1}\right], \ldots,\left[! A_{n}\right], \varsigma\left(! D_{1}, \ldots, ! D_{m}\right) \vdash ! B} P_{!} \frac{\frac{[\sigma]:\left[A_{1}\right], \ldots,\left[A_{n}\right], D_{1}, \ldots, D_{m} \vdash B}{\left[! A_{1}\right], \ldots,\left[! A_{n}\right], \varsigma\left(! D_{1}, \ldots, ! D_{m}\right) \vdash ! B} P_{!} \quad[\theta]: \Phi, \Gamma, ! B, ! B \vdash C}{\left[! A_{1}\right], \ldots,\left[! A_{n}\right],[! B \multimap ! B], \Phi, \varsigma\left(! D_{1}, \ldots, ! D_{m}\right), \Gamma, ! B \vdash C} L_{-}
\end{aligned}
$$

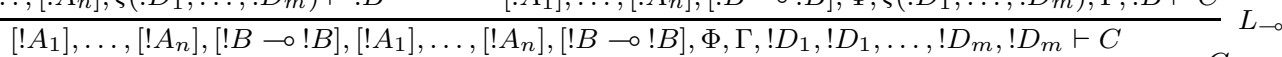

$$
\begin{aligned}
& \overline{\left[! A_{1}\right], \ldots,\left[! A_{n}\right],[! B \multimap ! B],\left[! A_{1}\right], \ldots,\left[! A_{n}\right],[! B \multimap ! B], \Phi, ! D_{m}, \Gamma, ! D_{1}, ! D_{1}, \ldots, ! D_{m-1}, ! D_{m-1} \vdash C}{ }^{C}
\end{aligned}
$$$$
\frac{\vdots}{\left[! A_{1}\right], \ldots,\left[! A_{n}\right],[! B \multimap ! B],\left[! A_{1}\right], \ldots,\left[! A_{n}\right],[! B \multimap ! B], \Phi, \varpi\left(! D_{1}, \ldots, ! D_{m}, \Gamma\right) \vdash C} C
$$ 
Observe that:

$$
\begin{array}{lll}
{[\pi]:} & {\left[! A_{1}\right], \ldots,\left[! A_{n}\right],\left[! B_{1} \multimap ! B_{2}\right]_{P_{1}-C_{!}}, \Phi_{1}, \Gamma_{1} \vdash C} \\
{[\rho]:} & :\left[! A_{1}^{1}\right], \ldots,\left[! A_{n}^{1}\right],\left[! B_{1}^{1} \multimap ! B_{2}^{1}\right],\left[! A_{1}^{2}\right], \ldots,\left[! A_{n}^{2}\right],\left[! B_{1}^{2} \multimap ! B_{2}^{2}\right], \Phi_{1}, \Gamma_{1} \vdash C
\end{array}
$$

Now, consider $\iota \|\left(\llbracket \rho \rrbracket_{e c}^{1} ; \llbracket \mathrm{TA}_{E} \rrbracket\right)$ and $\iota^{\prime} \|\left(\llbracket \pi \rrbracket_{e c}^{1} ; \llbracket \mathrm{TA}_{E} \rrbracket\right)$, where $E$ is the conclusion of $\rho$ and $\pi$. It is easy to realize that $\llbracket \rho \rrbracket_{e c a}$ can be simulated by the $\llbracket \pi \rrbracket_{e c a}$, in such a way that

$$
\begin{array}{r}
\llbracket \rho \rrbracket_{e c a}=\llbracket \pi \rrbracket_{e c a}\left\{\cdot / \bullet^{a}(\mathrm{f}, \mu)_{! B_{i}},\left(t, m_{! B_{i}^{1}}\right) /\left(\mathrm{I}(t), m_{! B_{i}}\right),\left(t, m_{! B_{i}^{2}}\right) /\left(\mathrm{r}(t), m_{! B_{i}}\right),\right. \\
\left.\cdot / \bullet^{b}(\mathrm{f}, \mu) ! A_{i},\left(t, m_{! A_{i}^{1}}\right) /\left(\mathrm{I}(t), m_{! A_{i}}\right),\left(t, m_{! A_{i}^{2}}\right) /\left(\mathrm{r}(t), m_{! A_{i}}\right)\right\}
\end{array}
$$

where $\mu$ is a metavariable for either open or close. Observe that $a=1$ when $\mu=$ close and $i=1$ (a promotion in $\pi$ raises a slot), $a=0$ otherwise and $b=0$ ( $\iota$ does not raise any slot). But there is exactly one (f, close)! $B_{1}$ in $\llbracket \pi \rrbracket_{\text {eca }}$ : at most one for obvious reasons (the same move is not played twice), at least one from Proposition 4 (since strategies interpreting (pseudo)proofs are total). The thesis easily follows.

Lemma 8 (Digging) If $\pi \stackrel{N}{\rightsquigarrow}$, then $\mathcal{C}\left(\llbracket \pi \rrbracket_{\text {eca }}\right)=\mathcal{C}\left(\llbracket \rho \rrbracket_{\text {eca }}\right)+1$.

Proof. We only consider the case where the cut reduced in $\pi$ is the last rule of $\pi$. The other cases can be reduced to this one by an easy induction. With this hypothesis, $\pi$ is

$$
\frac{\frac{D_{1}, \ldots, D_{m} \vdash B}{\varsigma\left(! D_{1}, \ldots, ! D_{m}\right) \vdash ! B} P ! \quad \frac{\Gamma, ! ! B \vdash C}{\vartheta(\Gamma), ! B \vdash C}}{\varpi\left(! D_{1}, \ldots, ! D_{m}, \Gamma\right) \vdash C} U
$$

and $\rho$ is

$$
\begin{aligned}
& \frac{D_{1}, \ldots, D_{m} \vdash B}{! D_{1}, \ldots, ! D_{m} \vdash ! B} P_{!} \\
& \frac{\frac{!_{1}, \ldots, ! ! D_{1}, \ldots, D_{m} \vdash ! ! B}{\Gamma, ! ! D_{1}, \ldots, ! ! D_{m} \vdash C} \quad \Gamma, ! ! B \vdash C}{! D_{m}, \Gamma, ! D_{1}, \ldots, ! D_{m}} U \\
& \frac{\Gamma, ! ! D_{1}, \ldots, ! ! D_{m} \vdash C}{! D_{m}, \Gamma, ! ! D_{1}, \ldots, ! ! D_{m-1} \vdash C} N_{!} \\
& \frac{\vdots}{\frac{!_{2}, \ldots, ! D_{m}, \Gamma, ! ! D_{1} \vdash C}{\varpi\left(! D_{1}, \ldots, ! D_{m}, \Gamma\right) \vdash C} N_{!}} N_{!}
\end{aligned}
$$

Moreover, $[\pi]$ is

$$
\frac{\frac{\left[A_{1}\right], \ldots,\left[A_{n}\right], D_{1}, \ldots, D_{m} \vdash B}{\left[! A_{1}\right], \ldots,\left[! A_{n}\right], \varsigma\left(! D_{1}, \ldots, ! D_{m}\right) \vdash ! B} P_{!} \frac{\Phi, \Gamma, ! ! B \vdash C}{\Phi, \vartheta(\Gamma), ! B \vdash C}}{\left[! A_{1}\right], \ldots,\left[! A_{n}\right],[! B \multimap ! B], \Phi, \varpi\left(! D_{1}, \ldots, ! D_{m}, \Gamma\right) \vdash C} N_{!}
$$

and $[\rho]$ is

$$
\begin{gathered}
\frac{\left[A_{1}\right], \ldots,\left[A_{n}\right], D_{1}, \ldots, D_{m} \vdash B}{\left[! A_{1}\right], \ldots,\left[! A_{n}\right], ! D_{1}, \ldots, ! D_{m} \vdash ! B} P_{!} \\
\frac{\frac{\left[! ! A_{1}\right], \ldots,\left[! ! A_{n}\right], ! ! D_{1}, \ldots, ! ! D_{m} \vdash ! ! B}{} P_{!} \quad \Phi, \Gamma, ! ! B \vdash C}{\left[! ! A_{1}\right], \ldots,\left[! ! A_{n}\right],[! ! B \multimap ! ! B], \Phi, \Gamma, ! ! D_{1}, \ldots, ! ! D_{m} \vdash C} L_{\multimap} \\
\frac{\left[! ! A_{1}\right], \ldots,\left[! ! A_{n}\right],[! ! B \multimap ! ! B], \Phi, ! D_{m}, \Gamma, ! ! D_{1}, \ldots, ! ! D_{m-1} \vdash C}{\vdots} N_{!} \\
\frac{\left[! ! A_{1}\right], \ldots,\left[! ! A_{n}\right],[! ! B \multimap ! ! B], \Phi, ! D_{2}, \ldots, ! D_{m}, \Gamma, ! ! D_{1} \vdash C}{\left[! ! A_{1}\right], \ldots,\left[! ! A_{n}\right],[! ! B \multimap ! ! B], \Phi, \varpi\left(! D_{1}, \ldots, ! D_{m}, \Gamma\right) \vdash C} N_{!}
\end{gathered}
$$


Observe that:

$$
\begin{array}{ll}
{[\pi] \quad:} & {\left[! A_{1}\right], \ldots,\left[! A_{n}\right],\left[! B_{1} \multimap ! B_{2}\right]_{P_{!}-N_{!}}, \Phi_{1}, \Gamma_{1} \vdash C} \\
{[\rho]:} & :\left[! A_{1}\right], \ldots,\left[! ! A_{n}\right],\left[! ! B_{1} \multimap ! ! B_{2}\right], \Phi_{1}, \Gamma_{1} \vdash C
\end{array}
$$

Now, consider $\iota \|\left(\llbracket \rho \rrbracket_{e c}^{1} ; \llbracket \mathrm{TA}_{E} \rrbracket\right)$ and $\iota^{\prime} \|\left(\llbracket \pi \rrbracket_{e c}^{1} ; \llbracket \mathrm{TA}_{E} \rrbracket\right)$, where $E$ is the conclusion of $\rho$ and $\pi$. It is easy to realize that $\llbracket \rho \rrbracket_{e c a}$ can be simulated by the $\llbracket \pi \rrbracket_{e c a}$, in such a way that

$$
\begin{array}{r}
\llbracket \rho \rrbracket_{e c a}=\llbracket \pi \rrbracket_{e c a}\left\{\cdot / \bullet^{a}(\mathrm{f}, \mu)_{! B_{i}},\left(t,\left(s, m_{B_{i}}\right)\right) /\left(\mathrm{n}(t, s), m_{B_{i}}\right),(t, \mu)_{! ! B_{i}} /(\mathrm{p}(t), \mu)_{! B_{i}},\right. \\
\left.\cdot / \bullet^{b}(\mathrm{f}, \mu) ! A_{i},\left(t,\left(s, m_{A_{i}}\right)\right) /\left(\mathrm{n}(t, s), m_{A_{i}}\right),(t, \mu)_{! ! A_{i}} /(\mathrm{p}(t), \mu) ! A_{i}\right\}
\end{array}
$$

where $\mu$ is a metavariable for either open or close. Observe that $a=1$ when $\mu=$ close and $i=1$ (a promotion in $\pi$ raises a slot), $a=0$ otherwise and $b=0$ ( $\iota$ does not raise any slot). But there is exactly one (f, close) $!_{1}$ in $\llbracket \pi \rrbracket_{e c a}$ : at most one for obvious reasons (the same move is not played twice), at least one from Proposition 4 (since strategies interpreting (pseudo)proofs are total). The thesis easily follows.

Lemma 9 (Weakening) If $\pi \stackrel{W}{w}$, then $\mathcal{C}\left(\llbracket \pi \rrbracket_{\text {eca }}\right)=\mathcal{C}\left(\llbracket \rho \rrbracket_{\text {eca }}\right)+1$.

Proof. We only consider the case where the cut reduced in $\pi$ is the last rule of $\pi$. The other cases can be reduced to this one by an easy induction. With this hypothesis, $\pi$ is

$$
\frac{\frac{D_{1}, \ldots, D_{m} \vdash B}{\varsigma\left(! D_{1}, \ldots, ! D_{m}\right) \vdash ! B} P_{!} \frac{\Gamma \vdash C}{\vartheta(\Gamma), ! B \vdash C}}{\varpi\left(! D_{1}, \ldots, ! D_{m}, \Gamma\right) \vdash C} U
$$

and $\rho$ is

$$
\begin{gathered}
\frac{\Gamma \vdash C}{\frac{! D_{m}, \Gamma \vdash C}{\vdots}} W \\
\frac{!_{D_{2} \ldots, ! D_{m}, \Gamma \vdash C}}{\varpi\left(! D_{1}, \ldots, ! D_{m}, \Gamma\right) \vdash C} W \\
W
\end{gathered}
$$

Moreover, $[\pi]$ is

$$
\frac{\frac{\left[A_{1}\right], \ldots,\left[A_{n}\right], D_{1}, \ldots, D_{m} \vdash B}{\left[! A_{1}\right], \ldots,\left[! A_{n}\right], \varsigma\left(! D_{1}, \ldots, ! D_{m}\right) \vdash ! B} P ! \frac{\Phi, \Gamma \vdash C}{\Phi, \vartheta(\Gamma), ! B \vdash C}}{\left[! A_{1}\right], \ldots,\left[! A_{n}\right],[! B \multimap ! B], \Phi, \varpi\left(! D_{1}, \ldots, ! D_{m}, \Gamma\right) \vdash C} L_{\multimap}
$$

and $[\rho]$ is

$$
\begin{gathered}
\frac{\Phi, \Gamma \vdash C}{\frac{\Phi, ! D_{m}, \Gamma \vdash C}{\vdots}} W \\
\frac{\Phi^{\Phi, ! D_{2} \ldots, ! D_{m}, \Gamma \vdash C}}{\Phi, \varpi\left(! D_{1}, \ldots, ! D_{m}, \Gamma\right) \vdash C} W \\
W
\end{gathered}
$$

Observe that:

$$
\begin{array}{ll}
{[\pi]:} & {\left[! A_{1}\right], \ldots,\left[! A_{n}\right],\left[! B_{1} \multimap ! B_{2}\right]_{P_{!}-W_{!}}, \Phi_{1}, \Gamma_{1}, \vdash C} \\
{[\rho]:} & \Phi_{1}, \Gamma_{1} \vdash C
\end{array}
$$

Now, consider $\iota \|\left(\llbracket \rho \rrbracket_{e c}^{1} ; \llbracket \mathrm{TA}_{E} \rrbracket\right)$ and $\iota^{\prime} \|\left(\llbracket \pi \rrbracket_{e c}^{1} ; \llbracket \mathrm{TA}_{E} \rrbracket\right)$, where $E$ is the conclusion of $\rho$ and $\pi$. It is easy to realize that $\llbracket \rho \rrbracket_{e c a}$ can be simulated by the $\llbracket \pi \rrbracket_{e c a}$, in such a way that

$$
\llbracket \rho \rrbracket_{e c a}=\llbracket \pi \rrbracket_{e c a}\left\{\cdot / \bullet^{a}(\mathrm{f}, \mu)_{! B_{i}}, \cdot / \bullet^{b}(\mathrm{f}, \mu)_{! A_{i}}\right\}
$$


where $\mu$ is a metavariable for either open or close. Observe that $a=1$ when $\mu=$ close and $i=1$ (a promotion in $\pi$ raises a slot), $a=0$ otherwise and $b=0$ ( $\iota$ does not raise any slot). But there is exactly one $(\mathrm{f}, \text { close })_{B_{1}}$ in $\llbracket \pi \rrbracket_{\text {eca }}$ : at most one for obvious reasons (the same move is not played twice), at least one from Proposition 4 (since strategies interpreting (pseudo)proofs are total). The thesis easily follows.

Lemma 10 (Box Commutation) If $\pi \stackrel{!-!}{\longleftrightarrow} \rho$, then $\mathcal{C}\left(\llbracket \pi \rrbracket_{e c a}\right)=\mathcal{C}\left(\llbracket \rho \rrbracket_{e c a}\right)+1$.

Proof. We only consider the case where the cut reduced in $\pi$ is the last rule of $\pi$. The other cases can be reduced to this one by an easy induction. (Since we are considering the reduction relation $\hookrightarrow$, we can assume $\pi$ to contain !-!-cuts, only.) With this hypothesis, $\pi$ is

$$
\frac{\frac{\sigma: A_{1}, \ldots, A_{n} \vdash B}{\varsigma\left(! A_{1}, \ldots, ! A_{n}\right) \vdash ! B} P_{!} \frac{\theta: \vartheta\left(B_{1}, \ldots, B_{m}, B\right) \vdash C}{! B_{1}, \ldots, ! B_{m}, ! B \vdash ! C}}{\varpi\left(! A_{1}, \ldots, ! A_{n}, ! B_{1}, \ldots, ! B_{m}\right) \vdash ! C} P_{!}
$$

and $\rho$ is

$$
\frac{\sigma: A_{1}, \ldots, A_{n} \vdash B \quad \theta: \varphi\left(B_{1}, \ldots, B_{m}\right), B \vdash C}{\frac{\vartheta\left(B_{1}, \ldots, B_{m},\left\langle A_{1}, \ldots, A_{n}\right\rangle\right) \vdash C}{\varpi\left(! A_{1}, \ldots, ! A_{n}, ! B_{1}, \ldots, ! B_{m}\right) \vdash ! C} P_{!}} U
$$

where $\vartheta\left(B_{1}, \ldots, B_{m},\left\langle A_{1}, \ldots, A_{n}\right\rangle\right)$ is $B_{\vartheta^{-1}(1)}, \ldots, B_{\vartheta^{-1}(k-1)}, A_{1}, \ldots, A_{n}, B_{\vartheta^{-1}(k+1)}, \ldots, B_{\vartheta^{-1}(m)}$ if $\vartheta(m+1)=k$. Moreover, $[\pi]$ is

$$
\frac{[\sigma]: \Psi_{1}, A_{1}, \ldots, A_{n} \vdash B}{! \Psi_{1}, \varsigma\left(! A_{1}, \ldots, ! A_{n}\right) \vdash ! B} P_{!} \frac{[\theta]: \Psi_{2}, \vartheta\left(B_{1}, \ldots, B_{m}, B\right) \vdash C}{! \Psi_{1},[! B \multimap ! B], ! \Psi_{2}, \varpi\left(! A_{1}, \ldots, \ldots, A_{n}, ! B_{1}, \ldots, ! B_{m}\right) \vdash ! C} P_{!}
$$

and $[\rho]$ is

$$
\frac{[\sigma]: \Psi_{1}, A_{1}, \ldots, A_{n} \vdash B \quad[\theta]: \Psi_{2}, \varphi\left(B_{1}, \ldots, B_{m}\right), B \vdash C}{\frac{\Psi_{1},[B \multimap B], \Psi_{2}, \vartheta\left(B_{1}, \ldots, B_{m},\left\langle A_{1}, \ldots, A_{n}\right\rangle\right) \vdash C}{! \Psi_{1},[!(B \multimap B)], ! \Psi_{2}, \varpi\left(! A_{1}, \ldots, ! A_{n}, ! B_{1}, \ldots, ! B_{m}\right) \vdash ! C} P_{\multimap}}
$$

Observe that:

$$
\begin{array}{ll}
{[\pi]:} & \varsigma\left(\left[! B_{1} \multimap ! B_{2}\right]_{P_{!}-P_{!}}, \Phi_{1}\right), \Gamma_{1} \vdash C \\
{[\rho]:} & \varsigma\left(\left[!\left(B_{1} \multimap B_{2}\right)\right], \Phi_{1}\right), \Gamma_{1} \vdash C
\end{array}
$$

Now, consider $\iota \|\left(\llbracket \rho \rrbracket_{e c}^{1} ; \llbracket \mathrm{TA}_{E} \rrbracket\right)$ and $\iota^{\prime} \|\left(\llbracket \pi \rrbracket_{e c}^{1} ; \llbracket \mathrm{TA}_{E} \rrbracket\right)$, where $E$ is the conclusion of $\rho$ and $\pi$. It is easy to realize that $\llbracket \rho \rrbracket_{e c a}$ can be simulated by the $\llbracket \pi \rrbracket_{e c a}$, in such a way that $\llbracket \rho \rrbracket_{e c a}$ is a permutation of

$$
\llbracket \pi \rrbracket_{e c a}\left\{(t, \text { open }) !\left(B_{1} \multimap B_{2}\right) /(t, \text { open })_{B_{2}}\left(t, \text { open }_{!_{1}}\right),(t, \text { close }) !\left(B_{1} \multimap ! B_{2}\right) / \bullet^{a}(s, \text { close }) !_{B_{1}}(s, \text { close }) !_{B_{2}}\right\}
$$

where $\mu$ is a metavariable for either open or close. Observe that $a=1$. Moreover, the only $s$ matching the pattern in (1) is $f$, since $f$ is the only exponential signature played by $\llbracket T A_{E} \rrbracket$. But there is exactly one occurrence of the sequence $(s, \text { close })_{B_{1}}(s, \text { close })_{B_{2}}$ in $\llbracket \pi \rrbracket_{\text {eca }}$ : at most one for obvious reasons (the same move is not played twice), at least one from Proposition 4 (since strategies interpreting (pseudo)proofs are total). The thesis easily follows.

\subsection{Full Abstraction}

We now have all the required material to state and prove our key result: full abstraction of the game model with respect to the reduction length (Theorems 2 and 3).

Given a proof $\pi$ and any reduction relation $\rightarrow,[\pi] \rightarrow$ and $\|\pi\| \rightarrow$ denote the maximum length of a reduction sequence starting in $\pi$ (under $\rightarrow$ ) and the maximum size of any reduct of $\pi$ (under $\rightarrow)$, respectively. We note $|\pi|$ the size of a proof $\pi$. 
Lemma 11 For every proof $\pi,[\pi]_{\rightsquigarrow}=[\pi]_{\hookrightarrow}$ and $\|\pi\|_{\rightsquigarrow}=\|\pi\|_{\hookrightarrow}$.

Proof. Whenever $\pi \stackrel{-!}{\sim} \rho \stackrel{x}{\rightsquigarrow} \sigma$ and $x \neq !-!$, there are $\theta_{1}, \ldots, \theta_{n}$ (where $n \geq 1$ ) such that $\pi \stackrel{x_{1}}{\rightsquigarrow} \theta_{1} \stackrel{x_{2}}{\rightsquigarrow} \ldots \stackrel{x_{n}}{\rightsquigarrow} \theta_{n} \stackrel{x_{n+1}}{\rightsquigarrow} \sigma$, and $x_{i+1}=!-$ ! whenever $x_{i}=$ !-!. For example, if $\pi \stackrel{!-!}{\rightsquigarrow} \rho \stackrel{W}{\rightsquigarrow} \sigma$ and the box erased in the second step is exactly the one created by the first step, then clearly $\pi \stackrel{W}{\rightsquigarrow} \underset{W}{W}$. As a consequence, for any sequence $\pi_{1} \rightsquigarrow \cdots \rightsquigarrow \pi_{n}$ there is another sequence $\rho_{1} \rightsquigarrow \cdots \rightsquigarrow \rho_{m}$ such that $\pi_{1}=\rho_{1}, \pi_{n}=\rho_{m}$ and $m \geq n$. This proves the first claim. Now, observe that for any $1 \leq i \leq n$ there is $j$ such that $\left|\rho_{j}\right| \geq\left|\pi_{i}\right|:$ a simple case analysis suffices. This concludes the proof.

Proposition 6 If $\pi \stackrel{\{\mathcal{C}, \mathcal{D}, \mathcal{N}, \mathcal{W}, !-!\}}{\hookrightarrow} \rho$ then $\mathcal{C}(\pi)=\mathcal{C}(\rho)+1$.

Proof. From Remark 1, we know that $\mathcal{C}(\pi)=\mathcal{C}(\llbracket \pi \rrbracket$ eca $)$. But by Lemmas $6,7,8,9$ we obtain the thesis.

Proposition 7 If $\pi \stackrel{\{\mathcal{T}, \mathcal{X}, \otimes,-\infty\}}{\longrightarrow} \rho$ then $\mathcal{C}(\pi)=\mathcal{C}(\rho)$.

Proof. We can proceed exactly as for Proposition 6.

Lemma 12 If $\pi$ is cut-free, then $\mathcal{C}(\pi) \leq|\pi|$.

Proof. An easy induction on $\pi$.

Proposition 8 If $\pi$ rewrites to $\rho$ in $n$ steps by the $\mathcal{T}$ rule, then $|\pi|=|\rho|$ and $n \leq 2|\pi|^{2}$.

Proof. The equality $|\pi|=|\rho|$ can be trivially verified whenever $\pi \stackrel{\mathcal{I}}{\hookrightarrow} \rho$. Now, for any proof $\pi$, define $|\pi|_{\text {comm }}$ as the the sum, over all instances of the $U$ rule inside $\pi$, of $|\sigma|_{\text {cut }}+|\sigma|+|\theta|$, where $\sigma$ (respectively, $\theta$ ) is the left (respectively, right) premise of the cut and $|\sigma|_{\text {cut }}$ is simply the number of instances of the cut rule in $\sigma$. For obvious reasons, $0 \leq|\pi|_{\text {comm }} \leq 2|\pi|^{2}$. Moreover, if $\pi \stackrel{\mathcal{I}}{\hookrightarrow} \rho$, then $|\pi|_{\text {comm }}>|\rho|_{\text {comm }}$. For example, consider the following commutative reduction step:

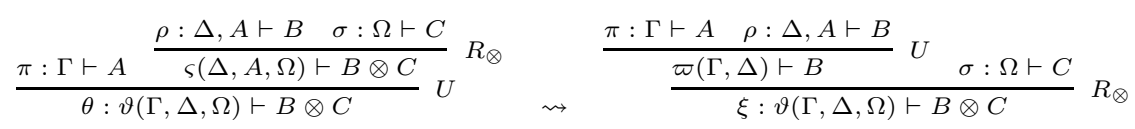

It is easy to see that $|\theta|=|\xi|$ but that $|\theta|_{\text {comm }}>|\xi|_{\text {comm }}$. Indeed:

$$
|\pi|_{\text {cut }}+|\pi|+|\rho|+|\sigma|+1>|\pi|_{\text {cut }}+|\pi|+|\rho| .
$$

Another interesting case is the following:

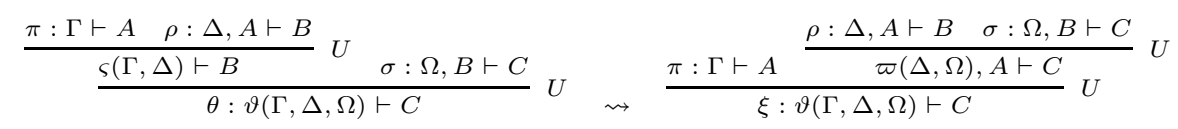

Again, $|\theta|=|\xi|$ but $|\theta|_{\text {comm }}>|\xi|_{\text {comm }}$. Other cases are similar. This concludes the proof.

Theorem 2 For every proof $\pi, \mathcal{C}(\pi) \leq[\pi]_{\rightsquigarrow}+\|\pi\|_{\rightsquigarrow}$

Proof. An easy consequence of Proposition 6, Proposition 7 and Lemma 12

Theorem 3 There is a polynomial $p: \mathbb{N} \times \mathbb{N} \rightarrow \mathbb{N}$ such that for every proof $\pi,[\pi]_{\rightsquigarrow},\|\pi\|_{\rightsquigarrow \leq} \leq$ $p(\mathcal{C}(\pi),|\pi|)$. 
Proof. By Lemma 11, the thesis easily follows from $[\pi]_{\hookrightarrow},\|\pi\|_{\hookrightarrow} \leq p(\mathcal{C}(\pi),|\pi|)$. Our first task will be to analyze the shape of any box you can find during the normalization of $\pi$ by $\hookrightarrow$ up to the point where you begin to fire !-! cuts. But it is easy to prove that any such box is just a subproof of $\pi$, possibly endowed with $n$ promotions rules (where $n$ is less than the total number of

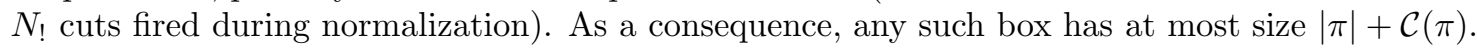
Now, we can easily bound $\|\pi\|_{\hookrightarrow}$ : at any $C$ or $N$ normalization step, the size of the underlying proof increases by at most $|\pi|+\mathcal{C}(\pi)$ (but the complexity strictly decreases), while in any other case the size decreases. As a consequence, $\|\pi\|_{\hookrightarrow} \leq \mathcal{C}(\pi)(|\pi|+\mathcal{C}(\pi))$. Now, the total number of non-commuting reduction steps is at most $\mathcal{C}(\pi)+\mathcal{C}(\pi)(|\pi|+\mathcal{C}(\pi))$. Between any of them, there are at most $2\|\pi\|_{\hookrightarrow}^{2}$ commuting steps. As a consequence:

$$
\begin{aligned}
{[\pi]_{\hookrightarrow} } & \leq \mathcal{C}(\pi)+\mathcal{C}(\pi)(|\pi|+\mathcal{C}(\pi))+(\mathcal{C}(\pi)+\mathcal{C}(\pi)(|\pi|+\mathcal{C}(\pi))) 2|| \pi \|_{\hookrightarrow}^{2} \\
& =(\mathcal{C}(\pi)+\mathcal{C}(\pi)(|\pi|+\mathcal{C}(\pi)))\left(1+2\|\pi\|_{\hookrightarrow}^{2}\right) \\
& \leq(\mathcal{C}(\pi)+\mathcal{C}(\pi)(|\pi|+\mathcal{C}(\pi)))\left(1+2(\mathcal{C}(\pi)(|\pi|+\mathcal{C}(\pi)))^{2}\right) .
\end{aligned}
$$

This concludes the proof.

\section{Further Work}

The main defect of our approach is the strong use of sequentiality information from sequent calculus proofs in the game interpretation. The two main approaches to get rid of this sequentiality are the use of non-deterministic strategies or of clusters of moves (when interpreting the promotion rule). This way we would be able to directly interpret proof-nets. In a similar spirit, we have used an exponential construction for games based on a grammar of exponential signatures, as usually done with context semantics. This is known to lead to not-very-satisfactory properties for !: for example, weakening is not neutral with respect to contraction, contraction is not commutative, etc. However, an answer to this problem should easily come from the solution proposed in the AJM setting with the notion of equivalence of strategies [2]. All these ingredients would probably allow us to turn our game model into a true categorical model of intuitionistic linear logic.

Another weakness is the restriction to surface reduction. We think adaptations to head reduction or to reduction strategies leading to normal forms should be possible by modifying the time analyzer in order to interactively access to "deeper" parts of proofs.

The notion of realizability we have introduced is tuned to reach the result we need, namely Proposition 4. However, it seems possible to modify it in various ways and to use it for very different applications in the more general context of game semantics.

Very recently, another proposal leading to similar observations but being based on relational semantics has appeared [6]. Possible correspondences between our game-theoretical analysis and the analysis done in [6] could come from works about projecting strategies into relations.

\section{References}

[1] Samson Abramsky. Semantics of interaction. In Peter Dybjer and Andrew Pitts, editors, Semantics and Logics of Computation, Publications of the Newton Institute, pages 1-32. Cambridge University Press, 1997.

[2] Samson Abramsky, Radha Jagadeesan, and Pasquale Malacaria. Full abstraction for PCF. Information and Computation, 163(2):409-470, December 2000.

[3] Patrick Baillot. Approches dynamiques en sémantique de la logique linéaire : jeux et géométrie de l'interaction. Thèse de doctorat, Université Aix-Marseille II, 1999.

[4] Patrick Baillot and Marco Pedicini. Elementary complexity and geometry of interaction. Fundamenta Informaticae, 45(1-2):1-31, 2001. 
[5] Ugo Dal Lago. Context semantics, linear logic and computational complexity. In Proc. 21st Symposium on Logic in Computer Science, pages 169-178. IEEE, 2006.

[6] Daniel de Carvalho, Michele Pagani, and Lorenzo Tortora de Falco. A semantic measure of the execution time in linear logic. Technical Report 6441, INRIA, 2007.

[7] Dan Ghica. Slot games: A quantitative model of computation. In Proc. 32nd ACM Symposium on Principles of Programming Languages, pages 85-97, 2005.

[8] Guy McCusker. Games and Full Abstraction for a Functional Metalanguage with Recursive Types. Ph.D. thesis, Imperial College and University of London, 1996. Published in SpringerVerlag's Distinguished Dissertations in Computer Science series, 1998. 